ARTICLE

\title{
Production of itaconic acid from alkali pretreated lignin by dynamic two stage bioconversion
}

Joshua R. Elmore (10) 1,2, Gara N. Dexter ${ }^{1}$, Davinia Salvachúa ${ }^{3}$, Jessica Martinez-Baird ${ }^{1}$, E. Anne Hatmaker (iD ${ }^{1}$, Jay D. Huenemann ${ }^{1,4}$, Dawn M. Klingeman ${ }^{1}$, George L. Peabody V1', Darren J. Peterson ${ }^{3}$, Christine Singer ${ }^{3}$, Gregg T. Beckham (10 ${ }^{3}$ \& Adam M. Guss (i) ${ }^{1,4 凶}$

Expanding the portfolio of products that can be made from lignin will be critical to enabling a viable bio-based economy. Here, we engineer Pseudomonas putida for high-yield production of the tricarboxylic acid cycle-derived building block chemical, itaconic acid, from model aromatic compounds and aromatics derived from lignin. We develop a nitrogen starvationdetecting biosensor for dynamic two-stage bioproduction in which itaconic acid is produced during a non-growth associated production phase. Through the use of two distinct itaconic acid production pathways, the tuning of TCA cycle gene expression, deletion of competing pathways, and dynamic regulation, we achieve an overall maximum yield of $56 \%(\mathrm{~mol} / \mathrm{mol})$ and titer of $1.3 \mathrm{~g} / \mathrm{L}$ from $p$-coumarate, and $1.4 \mathrm{~g} / \mathrm{L}$ titer from monomeric aromatic compounds produced from alkali-treated lignin. This work illustrates a proof-of-principle that using dynamic metabolic control to reroute carbon after it enters central metabolism enables production of valuable chemicals from lignin at high yields by relieving the burden of constitutively expressing toxic heterologous pathways.

\footnotetext{
${ }^{1}$ Biosciences Division, Oak Ridge National Laboratory, Oak Ridge, TN, USA. ${ }^{2}$ Biological Sciences Division, Pacific Northwest National Laboratory, Richland, WA, USA. ${ }^{3}$ National Bioenergy Center, National Renewable Energy Laboratory, Golden, CO, USA. ${ }^{4}$ Bredesen Center for Interdisciplinary Research, University of Tennessee, Knoxville, TN, USA. ${ }^{凶}$ email: gussam@ornl.gov
} 
$\mathrm{V}$ alorization of lignin, a complex aromatic heteropolymer and the second most abundant component of terrestrial biomass, will be critical for the economic viability of lignocellulosic biorefineries ${ }^{1}$. Biological upgrading of lignin has been demonstrated with production of aromatic catabolic intermediates $^{2-7}$ and their derivatives ${ }^{8}$, as well as carbon storage compounds such as polyhydroxyalkanoates (PHAs) ${ }^{9,10}$ and lipids $^{11}$ (Fig. 1, red boxes). However, the sizes of individual chemical markets are typically at least an order of magnitude smaller when compared to fuel markets. With an estimated billion tons of plant biomass able to be sustainably grown in the United States for lignocellulosic biofuel production ${ }^{12}$, hundreds of millions of tons of lignin-rich feedstock could be available for valorization in the United States alone. Therefore, lignin will need to be converted into a wide array of products to avoid oversaturating individual chemical markets and to replace petroleumderived incumbent molecules.

To increase the portfolio of products that can be made from lignin, additional parts of metabolism will need to be targeted. The tricarboxylic acid (TCA) cycle is a potential source of valuable chemicals including succinate and citrate, but it has not yet been harnessed for lignin valorization. Indeed, TCA cycle-derived chemicals are ideal products for lignin valorization because aromatic carbon is typically funneled directly into this part of metabolism. Itaconic acid is an unsaturated dicarboxylic acid derived from cis-aconitate in the TCA cycle, with industrial uses including as an acrylate alternative and for the production of polymers. ${ }^{13}$ Itaconic acid has been produced industrially from simple sugars, primarily glucose, since the $1950 \mathrm{~s}^{14,15}$, and its potential to functionally replace several petroleum-derived commodity chemicals was highlighted by its selection as one of the top bio-based platform chemicals in several reports, including a 2004 United States Department of Energy report. ${ }^{16}$ However, the relatively high cost of sugars makes itaconic acid production expensive, limiting it to use as a specialty chemical. Using cheap and abundant feedstocks, such as lignin, has the potential to reduce production $\operatorname{costs}^{17}$ and enable much broader industrial use of itaconic acid.

The saprophytic bacterium Pseudomonas putida KT2440 is a microbe of industrial interest $\mathrm{t}^{18,19}$ due to its robust metabolism ${ }^{20}$ and tolerance to xenobiotics. ${ }^{21-24} \mathrm{P}$. putida also has the ability to tolerate and catabolize a wide-range of aromatic compounds ${ }^{25}$
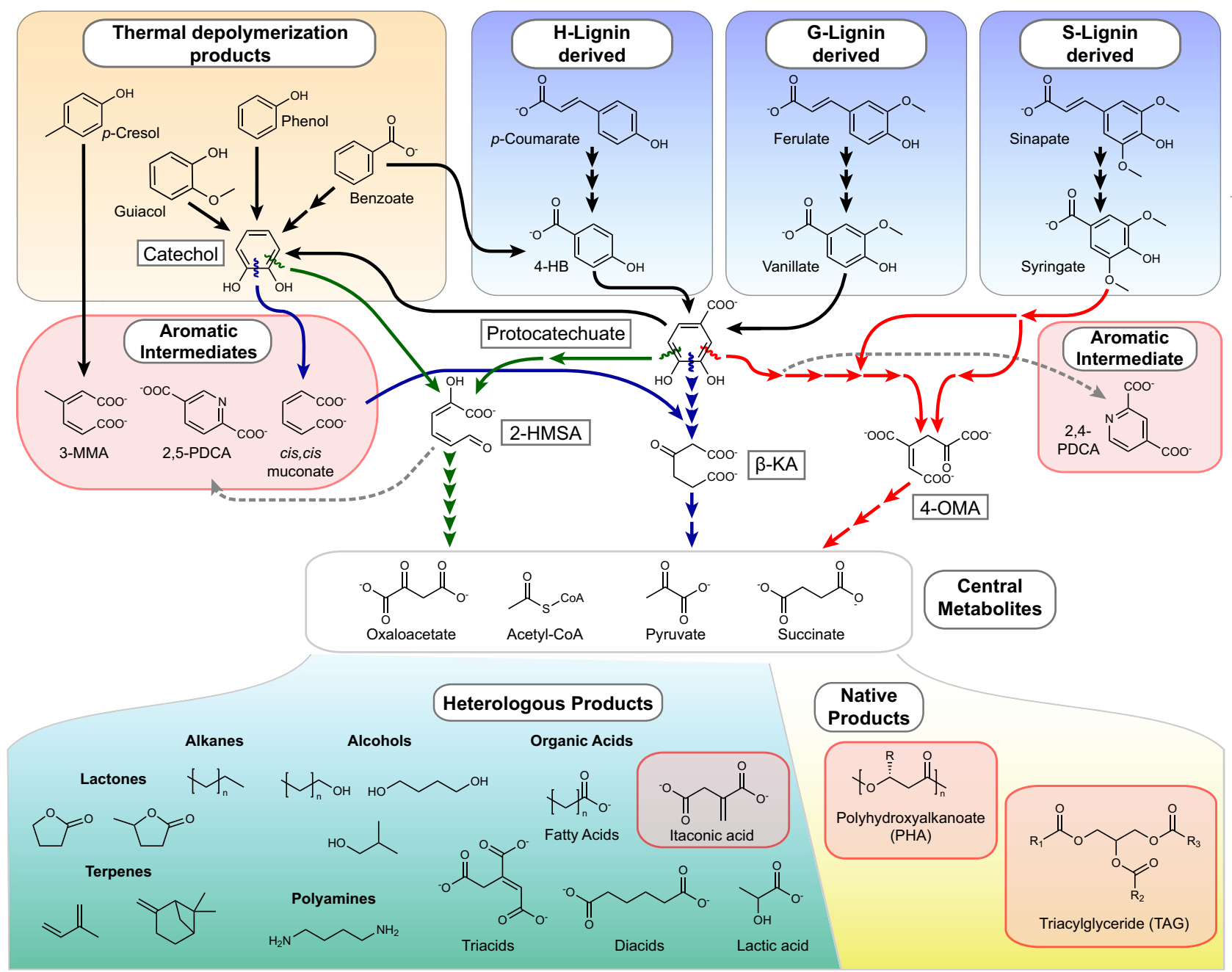

Fig. 1 Biological upgrading of lignin by funneling depolymerized lignin aromatics toward value added products up and downstream of central metabolism. Solid colored and black arrows indicate known metabolic pathway steps for conversion of aromatic intermediates into central metabolites, with dotted black arrows indicating predicted metabolic pathway steps. Dotted gray lines indicate heterologous pathways to convert aromatic intermediates to value-added aromatic derivatives. Red boxed compounds are those whose production from deconstructed lignin have been demonstrated, including itaconate from this study. Acronyms used above: 4-HB (4-hydroxybenzoate), 3-MMA (3-methylmuconate), 2,5-PDCA (2,5-pyridinedicarboxylate), 2,4PDCA (2,4-pyridinedicarboxylate), $\beta$-KA ( $\beta$-ketoadipate), 2-HMSA (2-hydroxymuconate semialdehyde), 4-OMA (4-oxalomesaconate). 

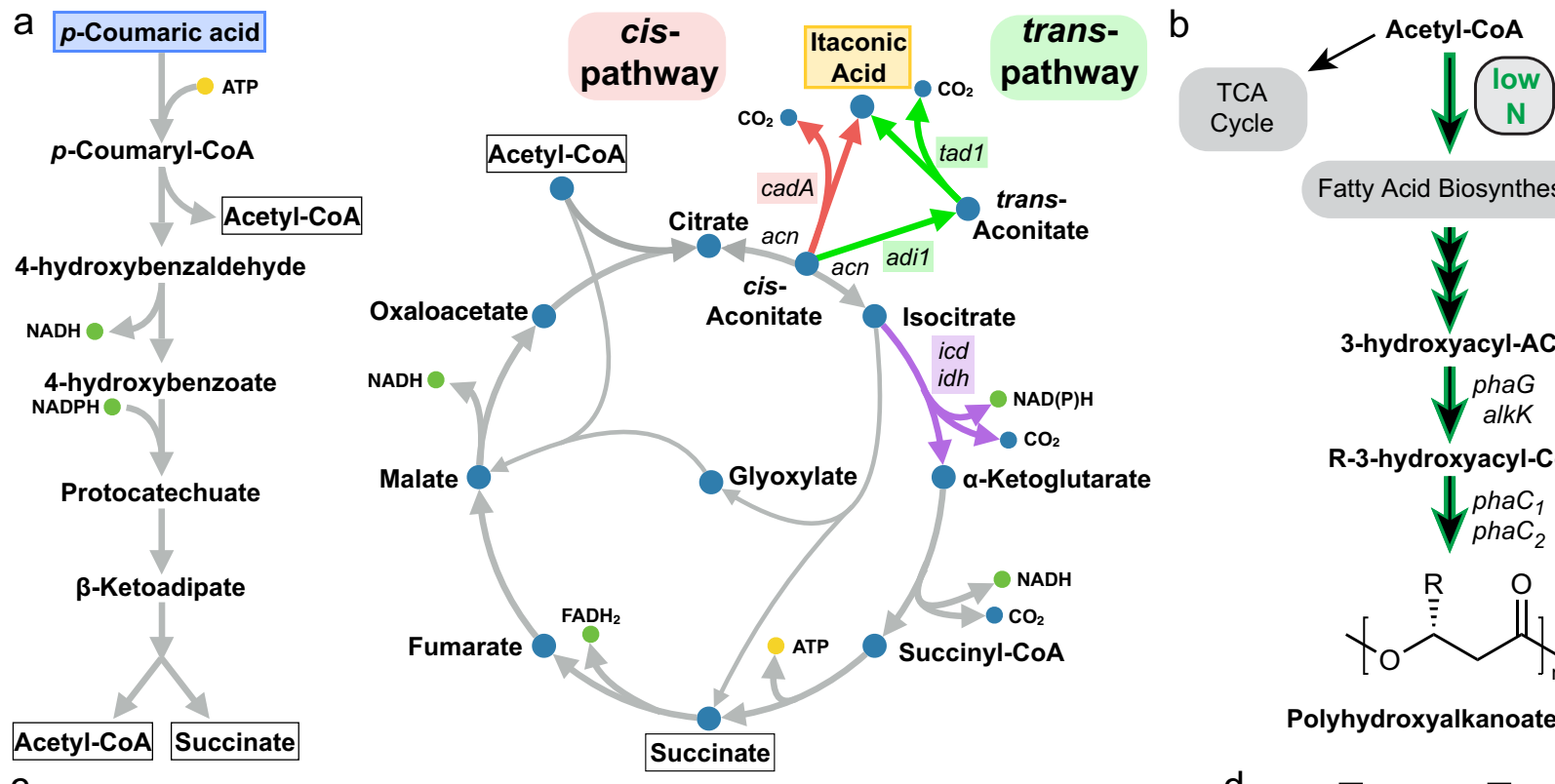

Fatty Acid Biosynthesis

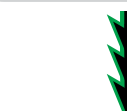

3-hydroxyacyl-ACP

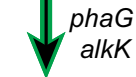

R-3-hydroxyacyl-CoA $\sqrt{\text { phac }_{1}}$<smiles>[R][C@H](CC(C)(C)C)OC(C)(C)C</smiles>

Polyhydroxyalkanoate (PHA)

C

JE4306 $\Delta p h a C_{1} Z_{2} i c d^{\mathrm{ATG}} i d h^{\mathrm{ATG}}$

JE4308 $\Delta p h a C_{1} Z_{2} i c d^{\mathrm{GTG}} i d h^{\mathrm{GTG}}$ $\Delta p h a C_{1} Z_{2} i c d^{T T G} i d h^{T T G}$
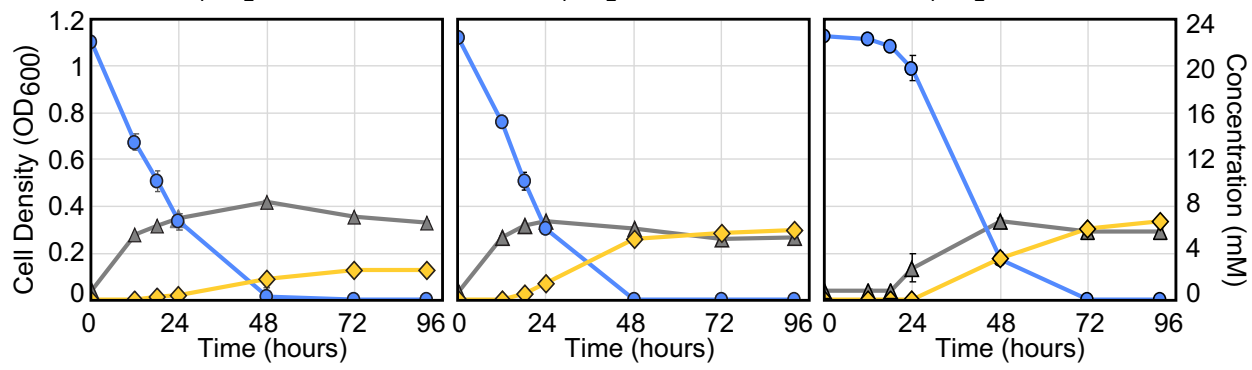

d

p-coumaric acid (mM)

$\diamond$ itaconic acid $(\mathrm{mM})$

$\triangle \mathrm{OD}_{600}$

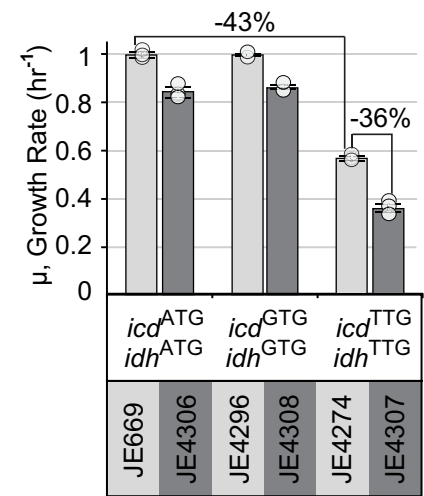

Fig. 2 Two-stage production of itaconic acid from $\boldsymbol{p}$-coumaric acid. a Simplified $p$-coumaric acid assimilation, $\beta$-ketoadipate, and tricarboxylic acid (TCA) cycle pathways in Pseudomonas putida KT2440 with modified or heterologous steps indicated by colored arrows, and connecting metabolites outlined. For simplicity some steps are not included. The cis (red arrow) and trans (green arrow) pathways for itaconic acid are indicated with involved genes, cadA (cis) $\&$ tad1/adi1 (trans) adjacent to the reaction their gene products perform. Isocitrate dehydrogenase activity, provided by the icd \& idh gene products, is indicated by a purple arrow. b Simplified polyhydroxyalkanoate (PHA) biosynthesis pathway in P. putida KT2440. The PHA pathway, via fatty acid biosynthesis, competes with the TCA cycle for acetyl-CoA during nitrogen-limited conditions. c Production of itaconic acid from $p$-coumaric acid in shake flasks by $P$. putida strains constitutively expressing cadA under nitrogen-limited conditions. Strain name and their unique modifications are indicated above the charts. Cell density (OD600, gray diamonds), residual $p$-coumaric acid (mM, blue circles), and produced itaconic acid (mM, yellow triangles) are indicated. $\mathbf{d}$ Growth rates of $P$. putida strains containing icd \& idh start codon mutations with or without constitutive cadA expression using $p$-coumaric acid as sole carbon source. Rates were determined by 48 -well microtiter plate cultivation. $\mathbf{c}, \mathbf{d}$ Data are represented as mean values \pm standard deviation in three replicates. Source data underlying Fig. $2 \mathrm{c}$ and $\mathrm{d}$ is provided as a Source Data file.

which led to its recent use in upgrading depolymerized lignin into PHAs $^{10,26}$, cis,cis-muconic acid 35,27 , and other intermediates in aromatic catabolism ${ }^{7}$. In P. putida, p-hydroxyphenyl $(\mathrm{H})$ and guiacyl (G) lignin-derived aromatics are funneled via the $\beta$ ketoadipate pathway to acetyl-CoA and succinate (Fig. 2a). This direct route to key TCA cycle intermediates suggests that high yields of TCA cycle-derived products such as itaconic acid should be possible from lignin. For instance, because the lignin-derived aromatic compound $p$-coumaric acid is catabolized into one succinate and two acetyl-CoA molecules, the theoretical maximum yield of itaconic acid is $1.33 \mathrm{~mol}$ itaconic acid $/ \mathrm{mol} p$-coumaric acid.

Growth phase production of itaconic acid may be challenging because itaconic acid disrupts bacterial growth via inhibition of enzymes in the glyoxylate shunt ${ }^{28}$ and citramalate cycle ${ }^{29}$. An alternate approach is to use a two-stage process to decouple growth of the microbial catalyst from conversion of feedstocks to chemicals, which provides solutions to many problems present in growth-associated processes (e.g., product toxicity, slow catalyst growth). ${ }^{30}$ Such processes often take advantage of the natural responses to nutrient limitations (e.g., nitrogen, sulfur, phosphate) and environmental shifts (e.g., $\mathrm{O}_{2}$ limitation, temperature shifts) that prevent microbial growth while maintaining the metabolic reactions of interest. Coupling two-stage processes with dynamic metabolic control has the potential to entirely remodel metabolism.

In this study, we engineer $P$. putida to produce a commercially relevant chemical at high yields and gram-per-liter titers from model aromatic substrates and corn stover-derived, alkalipretreated lignin. We further develop multiple production pathways and developed a signal-amplified biosensor for two-stage production via dynamic metabolic control to increase efficiency and mitigate toxicity. 


\section{Results}

Initial production of itaconic acid from aromatic compounds. The enzyme cis-aconitate decarboxylase, encoded by the Aspergillus terreus cadA gene ${ }^{31}$, produces itaconic acid by enzymatic decarboxylation of the TCA cycle intermediate cis-aconitate (Fig. 2a). Using a previously developed site-specific DNA integration system in $P$. putida $\mathrm{KT} 2440^{32}$, we integrated a constitutively expressed, codon optimized copy of cadA ( $\left.\mathrm{P}_{\text {tac }}: c a d A\right)$ into the genome of $P$. putida strain JE90 (named JE4305), and measured itaconic acid production from $p$-coumaric acid, the most abundant aromatic monomer released from corn stover during alkaline treatment ${ }^{3}$, as the sole carbon source. Under nitrogen-replete conditions, we were unable to detect itaconic acid production.

P. putida diverts carbon toward fatty acid biosynthesis for PHA production during nitrogen-starvation conditions (Fig. 2b). ${ }^{9,10}$ Thus, we hypothesized that more carbon would be directed to itaconic acid production under similar conditions. As predicted, nitrogen-starvation conditions enabled detectable itaconic acid production, but the yield was low $(4.2 \% \mathrm{~mol} / \mathrm{mol})$ (Fig. 2c; Table 1). Because PHA production competes for carbon, we deleted the PHA synthetases $p h a C_{1}$ and $p h a C_{2}$ (strain named JE4306), increasing overall itaconic acid yield by approximately 3fold $(12 \% \mathrm{~mol} / \mathrm{mol})$ (Fig. $2 \mathrm{c}$; Table 1$)$. However, the majority of itaconic acid was produced during stationary phase, supporting the notion that more carbon is directed to itaconic acid production during nitrogen-starvation. During the non-growing stationary phase from 24 to $96 \mathrm{~h}$, also referred to as the production phase, the itaconic acid yield from the remaining $p$ coumaric acid increased from undetectable production during growth phase to $33 \% \mathrm{~mol}$ itaconic acid $/ \mathrm{mol} p$-coumaric acid.

Modulating TCA cycle flux increases itaconic acid yields and titers. As an obligate aerobe, $P$. putida maintains robust TCA cycle activity for energy production. We hypothesized that reducing flux through isocitrate dehydrogenase (Fig. $2 \mathrm{a}-i c d, i d h$ ) should increase accumulation of cis-aconitate, and therefore increase yields. However, deletion of $i c d$ and $i d h$ would make $P$. putida an energy-starved, $\alpha$-ketoglutarate auxotroph, and likely unable to grow on lignin-derived substrates. Thus, we aimed to reduce the translation efficiency of $i c d$ and $i d h$, which are encoded next to each other on the chromosome and divergently transcribed. Using reporter gene fusions, we showed that changing the translational start codon of the fluorescent protein mNeonGreen to GTG or TTG reduced fluorescence per cell by 2.3- and 4.2 -fold, respectively (Supplementary Table 1), suggesting that this approach could be used to decrease the level of TCA cycle enzymes. Therefore, we altered the start codons of icd and idh such that they were each GTG or TTG, generating strains JE4296 (icd ${ }^{\mathrm{GTG}}: i d h^{\mathrm{GTG}}$ ) and JE4274 (icd TTG:idhTTG), respectively. Cell yield (as measured by final $\mathrm{OD}_{600}$ ) was largely unaffected by the start codon alterations (Supplementary Fig. 1a). The growth rate of JE4296 was also unaffected, while the growth rate of JE4274 on p-coumaric acid was decreased by $43 \%$ (Fig. 2 d, Supplementary Fig. 1a).

To determine the impact of these mutations on itaconic acid production, we integrated the $\mathrm{P}_{\text {tac }}: c a d A$ cassette into both strains, generating strains JE4308 (icd $\mathrm{GTG}_{: i d h \mathrm{GTG}} \mathrm{P}_{\text {tac }}: c a d A$ ) and JE4307 (icd TTG:idhTTG, $\mathrm{P}_{t a c}: c a d A$ ), and assayed itaconic acid production from $p$-coumaric acid under nitrogen-limited and nitrogen-replete conditions. Slowing the TCA cycle was sufficient to allow detectable itaconic acid production under nitrogen-replete conditions (Supplementary Fig. 1b), and nitrogen-limited conditions increased overall yields from 12 to $27 \%$ and $30 \% \mathrm{~mol} / \mathrm{mol}$ with JE4308 and JE4307, respectively (Fig. 2c; Table 1). During the non-

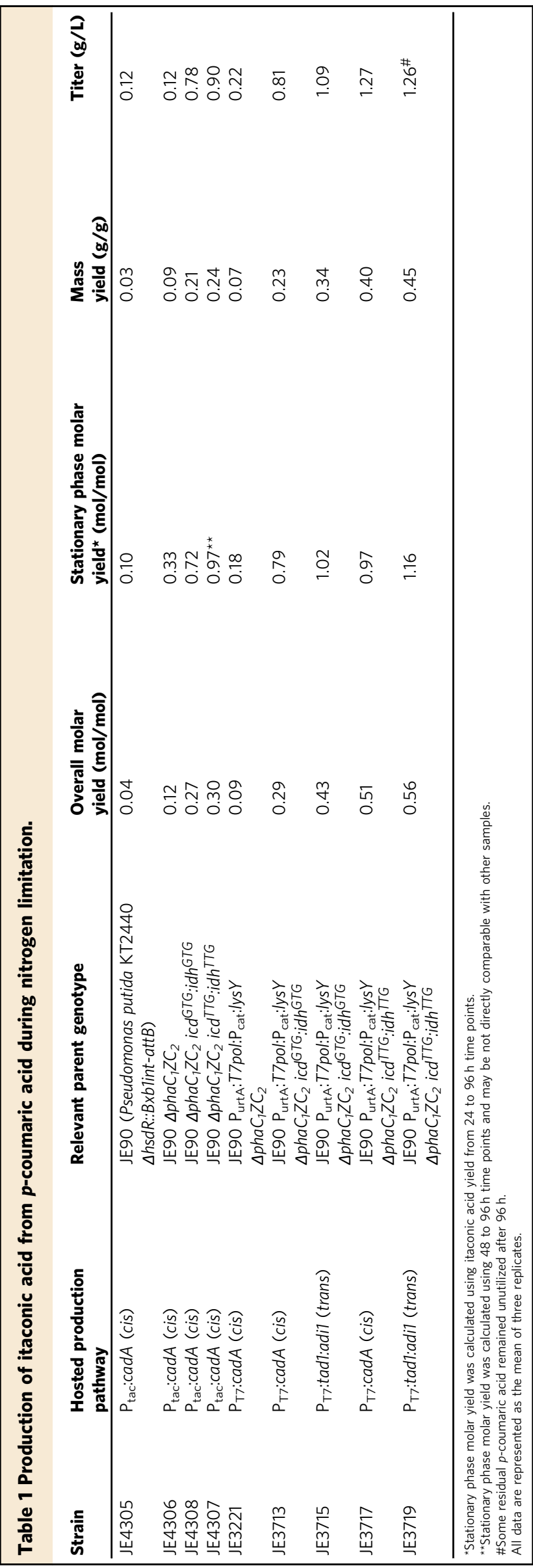


growing production phase, itaconic acid yield increased to $72 \%$ $\mathrm{mol} / \mathrm{mol}$ for strain JE4308 and 97\% for strain JE4307. While yields improved, the detrimental effect of constitutive cadA expression was highlighted by decreased growth rates in strains expressing cadA (Fig. 2d). Growth rates in all three genetic backgrounds were negatively impacted by constitutive cadA expression, suggesting that expression of cadA may be toxic. The impact of cadA expression was most pronounced in JE4307, where it caused a 36\% reduction in growth rate over parent strain JE4274.

Development of a signal-amplified nitrogen-limitation biosensor for dynamic metabolic control in P. putida KT2440.
Limiting the expression of the apparently toxic CadA protein to the production phase via dynamic regulation could substantially improve both growth and itaconic acid production. Therefore, we pursued development of an expression system that is induced upon nitrogen starvation. Furthermore, native regulatory systems are typically tuned to provide expression sufficient for associated pathways, which is often insufficient for heterologous pathways. However, use of an orthogonal RNA polymerase intermediary such as the T7 RNA polymerase (T7pol ${ }^{33}$ can allow amplification of the original signal ${ }^{34}$ (Fig. 3a).

In the absence of preferred nitrogen sources, such as ammonium, P. putida activates genes for both the nitrogen-

a
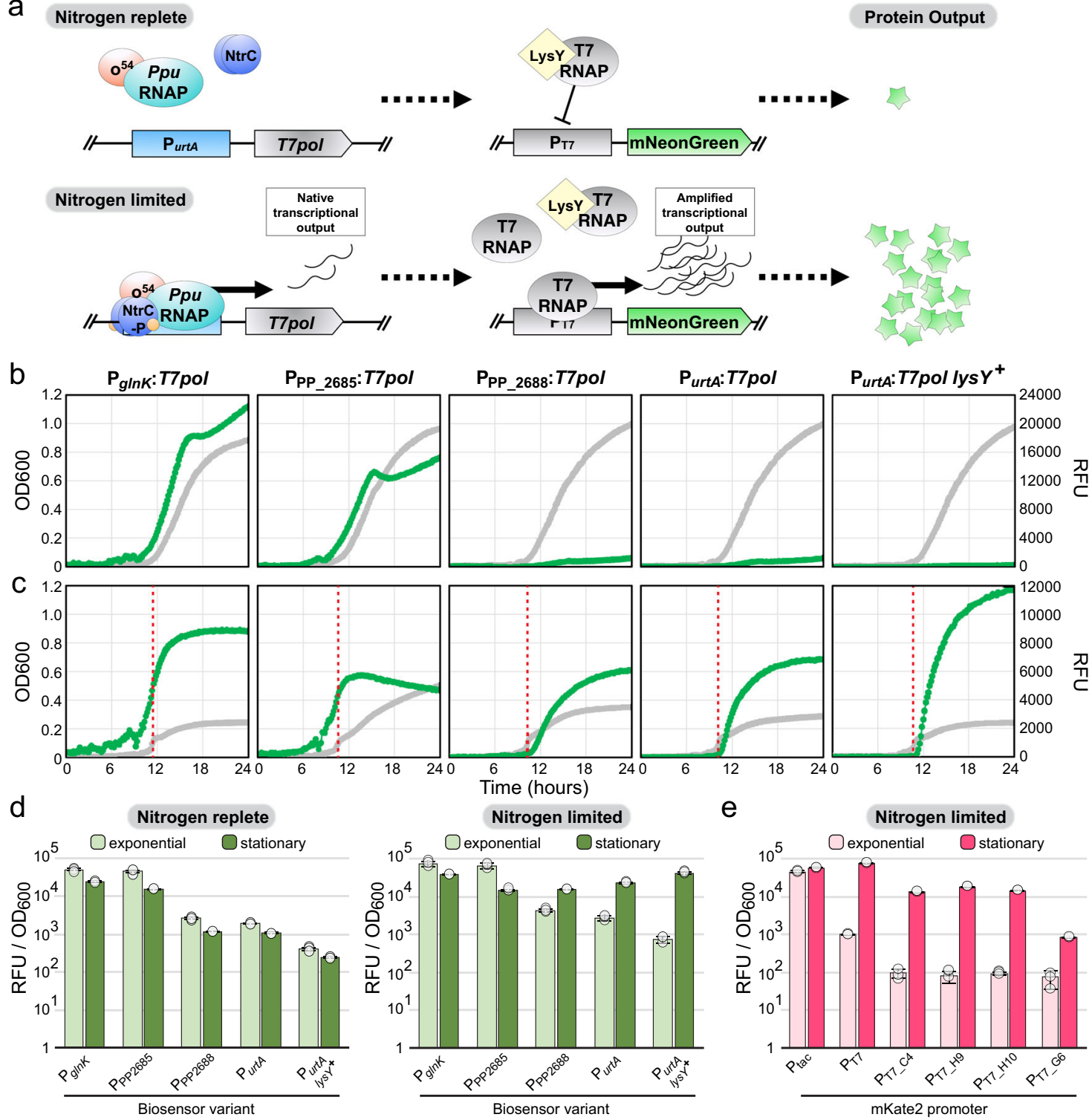

Fig. 3 Development of a nitrogen-limitation biosensor to enable dynamic control of two-stage bioproductions. a Diagram of biosensor design and utilization as a regulated signal amplifier for pathway and tool expression. P. putida RNA polymerase (Ppu RNAP), sigma factor 54 ( $\sigma 54$ ), T7 RNA polymerase gene (T7pol), wavy line (mRNA), T7 RNA polymerase protein (T7 RNAP), green stars (mNeonGreen protein). Representative growth curves of triplicate cultures for 96 -well microtiter plate cultivations of candidate biosensor strains, biosensor variant indicated, with integrated $\mathrm{P}_{T 7}$ : $\mathrm{mNeonGreen}$ cassette in either nitrogen-replete (b) or nitrogen-limited (c) medium. Cell density and mNeonGreen production, as measured by OD600 (gray) and relative fluorescence units (RFU-green) respectively, were measured every $10 \mathrm{~min}$. Entry to stationary phase is indicated (red dotted line) for nitrogenlimited cultures. d Graph of mNeonGreen production by candidate biosensor sensor strains during exponential growth (light green) or stationary phase (dark green) in microtiter plate cultivations. e Graph of mKate2 production by JE2113-derivatives with integrated $\mathrm{P}_{T 7}$-variant:mKate2 cassettes during exponential growth (light pink) or stationary phase (dark pink) in plate reader cultivations. d, e Data are presented as the mean values \pm standard deviation in three replicates. Source data underlying Fig. 3b-e is provided as a Source Data file. 
Table 2 Differential expression of genes downstream of potential nitrogen-sensitive promoters in P. putida JE1657.

\begin{tabular}{|c|c|c|c|c|}
\hline Locus Tag & Gene Name & $\log _{2}$ fold change $\left(\mathrm{NaNO}_{3} / \mathrm{NH}_{4} \mathrm{Cl}\right)$ & Base mean & Predicted gene function \\
\hline PP_1705 & nirB & 8.14 & 2029.14 & nitrite reductase large subunit \\
\hline PP_2092 & nasA & 6.31 & 361.67 & nitrate transporter \\
\hline PP_2094 & nass & 2.79 & 51.23 & nitrate binding protein \\
\hline PP_2688 & - & 3.99 & 132.41 & circularly permuted ATP-grasp type 2 \\
\hline PP_2842 & ure $D$ & 4.38 & 181.83 & urease accessory protein \\
\hline PP_4053 & treY & 2.19 & 1151.28 & maltooligosyl trehalose synthase \\
\hline PP_4845 & urtE & 3.77 & 67.21 & $A B C$ transporter ATP-binding protein \\
\hline PP_5234 & $g \ln K$ & 1.45 & 8496.51 & NRII(GInL/NtrB) phosphatase activator \\
\hline
\end{tabular}

Values represent the output of DEseq2 software package using four replicates for each nitrogen source.

starvation response and utilization of alternative nitrogensources $^{35,36}$. To identify a nitrogen starvation-sensitive promoter in $P$. putida, we performed transcriptomics during growth with a preferred (ammonia) or alternative (nitrate) nitrogen source (Table 2 and Supplementary Table 2). Based on the combination of these results and previous studies ${ }^{35,36}$, we selected four candidate promoters to test as biosensors: $\mathrm{P}_{\mathrm{PP} \_2685}, \mathrm{P}_{\mathrm{PP} \_2688}$, $\mathrm{P}_{\text {urtA }}$, and $\mathrm{P}_{\text {glnK }}$. Candidate promoters were used to express T7pol and integrated into the JE90 genome along with the gene encoding fluorescent protein mNeonGreen under control of a T7 promoter. Strains were grown under nitrogen-replete and nitrogen-limited conditions (Fig. 3b-d). While the $\mathrm{P}_{g l n K}$ and $\mathrm{P}_{\mathrm{PP} \_2685}$ promoters were surprisingly nitrogen-agnostic, displaying constitutive mNeonGreen expression similar to the $\sigma^{70}$ tac promoter (Supplementary Fig. 2), the other candidates $\mathrm{P}_{\mathrm{PP} \_2688}$ and $\mathrm{P}_{\text {urta }}$ responded to nitrogen-limitation, demonstrating 3.7 and 8.8 -fold mNeonGreen induction upon entry into nitrogendepletion-induced stationary phase (Fig. 3d).

While the initial $\mathrm{P}_{\text {urtA }}$ biosensor variant allowed strong induced expression, basal expression in the presence of nitrogen was relatively high. To reduce basal T7pol activity, we constitutively expressed a catalytically-deactivated variant of T7 lysozyme $(\text { LysY })^{37}$, which allosterically inhibits T7pol activity ${ }^{38}$ (Fig. 3a). The expression of LysY substantially improved biosensor performance, decreasing basal $\mathrm{mNeonGreen} \mathrm{expression} \mathrm{by} 78 \%$ in exponential phase and increasing the maximal induced mNeonGreen expression level. This resulted in a 60-fold mNeonGreen induction, a 6.8 -fold improvement over the strain lacking LysY (Fig. 3d). As an orthogonal measurement of biosensor performance, we utilized RNAseq to compare T7pol gene expression in strain JE2212 ( $\mathrm{P}_{\text {urtA }}$ :T7pol:lys $Y+\mathrm{P}_{\mathrm{T} 7}$ :mNeonGreen) with ammonium or nitrate as the sole nitrogen source as done above. Highlighting the function of this biosensor as a signal amplifier, $\mathrm{NO}_{3}$-induced $m$ NeonGreen mRNA abundance was 302- and 54-fold higher than urtA and T7pol, respectively (Supplementary Data 1).

Optimal pathway performance often requires tuning expression of individual proteins. Tuning expression is often achieved with promoter ${ }^{32,39}$ and/or $\mathrm{RBS}^{40}$ modifications. We utilized a small library of $\mathrm{T} 7$ promoter variants ${ }^{39}$ with the red fluorescent protein mKate2 to tune the magnitude of biosensor outputs. Unlike the $\sigma^{70}$ tac promoter (Supplementary Fig. 2a) which was constitutively expressed, nitrogen-limitation was required for induction of mKate 2 production in all five T7 promoter variants (Fig. 3e, Supplementary Table 3). Within the promoter library, maximum protein expression levels varied over an 89 -fold range. Interestingly, we observed a 2-3.5-fold dynamic range improvement over the standard T7 promoter with three of the variant promoters-largely driven by considerably lower basal expression -which approached the background autofluorescence.

Dynamic regulation improves two-stage production of itaconic acid production from lignin-derived aromatics. We next tested whether dynamic regulation of $c a d A$ would improve itaconic acid production by combining the altered isocitrate dehydrogenase start codons and the $\mathrm{P}_{\text {urtA }}$ :T7pol:lys $Y^{+}$biosensor cassette. The original T7 promoter showed the highest maximal expression level, so we integrated cadA under the control of this T7 promoter into all three strains (named JE3221, JE3713, and 3717; Table 1), and assayed production of itaconic acid from $p$-coumaric acid under nitrogen-limited conditions. Similar to previous shake flask experiments-with the exception of JE4307 ( $\mathrm{P}_{t a c}:$ cadA, icd TTG: $i d h^{\mathrm{TTG}}$ )-growth is complete within the first $24 \mathrm{~h}$, with some itaconic acid production occurring, likely after growth is completed. Itaconic acid yields with JE3221 ( $\mathrm{P}_{\mathrm{T} 7}$ :cadA, $i c d^{\mathrm{ATG}}$ : $\left.i d h^{\mathrm{ATG}}\right)$ and JE3713 ( $\left.\mathrm{P}_{\mathrm{T} 7}: c a d A, i c d^{\mathrm{GTG}}: i d h^{\mathrm{GTG}}\right)$ were similar to their corresponding constitutive cadA strains (Figs. 2c, 4a). Strains JE3713 ( $\mathrm{P}_{\mathrm{T} 7}:$ cadA $)$ and JE4308 $\left(\mathrm{P}_{\mathrm{tac}}:\right.$ cadA $)$ also displayed similar yield from remaining $p$-coumaric acid in the non-growing production phase, 72 and $79 \% \mathrm{~mol} / \mathrm{mol}$, respectively. Strain JE3717 ( $\left.\mathrm{P}_{\mathrm{T} 7}: c a d A, i c d^{\mathrm{TTG}}: i d h^{\mathrm{TTG}}\right)$, on the other hand, achieved an overall itaconic acid yield of $51 \% \mathrm{~mol} / \mathrm{mol}$ (Fig. $4 \mathrm{a}$, Table 1 ), a $67 \%$ improvement over the best performing constitutive cadA expression strain (Fig. 2c). Furthermore, dynamic regulation of cadA eliminated the growth defect induced by cadA expression (Fig. 4c), which has ramifications on itaconic acid productivityat $48 \mathrm{~h}$ JE3717 $\left(\mathrm{P}_{\mathrm{T} 7}: c a d A\right.$, icd $\left.{ }^{\mathrm{TTG}}: i d h^{\mathrm{TTG}}\right)$ itaconic acid production is essentially complete (Fig. 4b), while production by JE4307 ( $\mathrm{P}_{\text {tac }}: c a d A, i c d^{\text {TTG }}: i d h^{\text {TTG }}$ ) was not complete after $72 \mathrm{~h}$ (Fig. 2c). Taken together, dynamic regulation of cadA improves performance, and it will likely improve strain stability by eliminating the growth defect.

Metabolic pathway selection to optimize itaconic acid production. To date, other than in some organisms that natively produce itaconic acid, attempts to engineer strains for itaconic acid production have focused on the heterologous expression of the cis-aconitate decarboxylase (termed here the cis-pathway) from A. terreus. However, an alternate pathway for itaconic acid production was recently discovered in Ustilago maydis. ${ }^{41}$ This pathway, referred to here as the trans-pathway, proceeds through two steps. First, cis-aconitate is isomerized by aconitate isomerase (adi1) to the thermodynamically favorable isomer trans-aconitate, which is subsequently decarboxylated by trans-aconitate decarboxylase $(\operatorname{tad} 1)$ to generate itaconic acid (Fig. 2a). The trans 
a
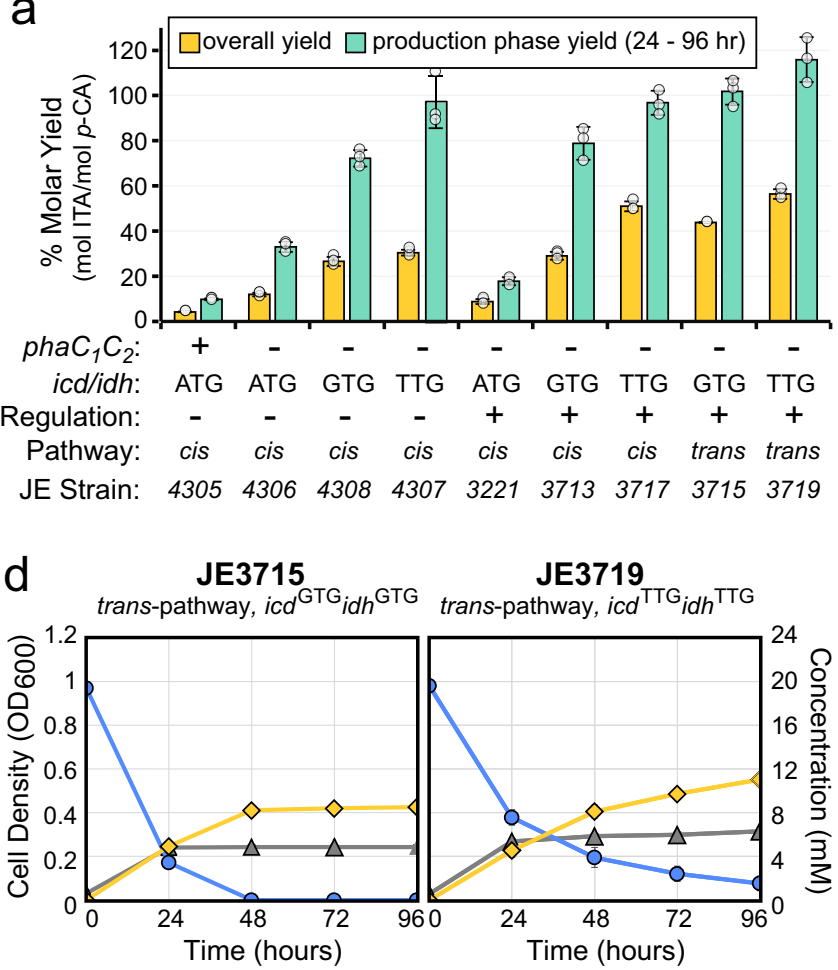

p-coumaric acid $(\mathrm{mM}) \diamond$ itaconic acid $(\mathrm{mM})$ b

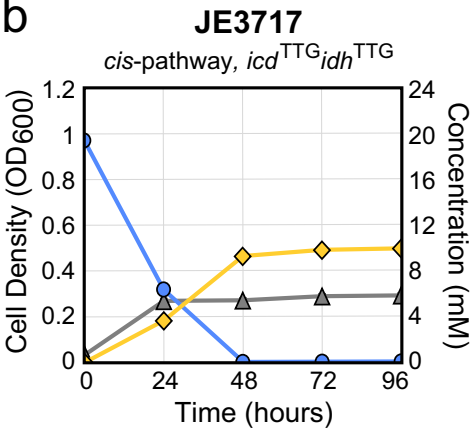

Op-coumaric acid (mM)

$\diamond$ itaconic acid $(\mathrm{mM})$
C

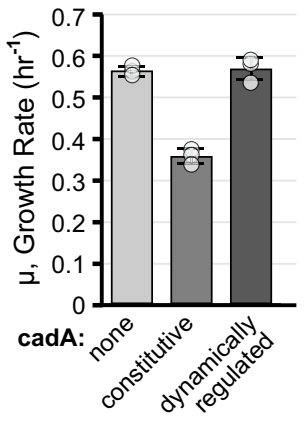

e

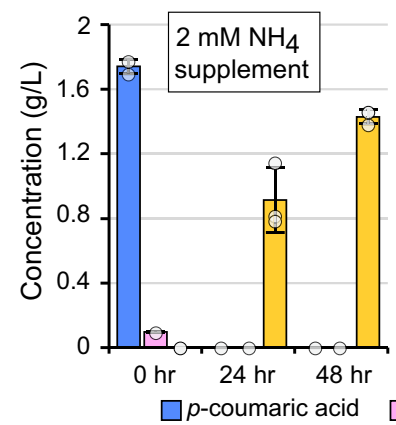

f

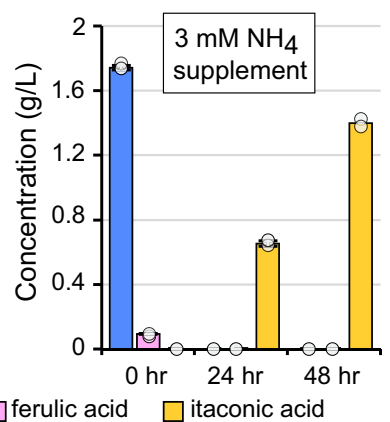

Fig. 4 Biosensor-controlled expression of itaconic acid production pathways enables high yield production from lignin and model aromatic substrates. a Molar yield of engineered strains from shake flask experiments with $20 \mathrm{mM} \mathrm{p}$-coumaric acid as sole carbon source. Overall yield (yellow) and production phase yield (green) are indicated. Production phase was defined as $24 \mathrm{hr}$ to $96 \mathrm{hr}$ time points. Relevant genetic modifications include the presence ( + ) or deletion (-) of the native PHA polymerase genes $\operatorname{phaC}_{1} C_{2}$, the start codon used (ATG, GTG, or TTG) for isocitrate dehydrogenase genes icd and idh, use of a constitutive $(-)$ or dynamically regulated $(+)$ itaconic acid biosynthesis pathway, the cis- or trans- itaconic acid production pathway, and the corresponding stain name. (b,d) Production of itaconic acid from $p$-coumaric acid in shake flasks by strains utilizing dynamically-regulated (b) cadA (cispathway) or (d) tad1/adi1 (trans-pathway) under nitrogen-limited conditions. Strain names and their unique modifications are indicated above the charts. Cell density (OD600, gray diamonds), p-coumaric acid (mM, blue circles), and itaconic acid (mM, yellow triangles) are indicated. c The effect of cadA expression on growth of $P$. putida icd ${ }^{\mathrm{TTG}} i \mathrm{dh}^{\mathrm{TTG}}$ strains in 48-well microtiter plate assays with $p$-coumaric acid as sole carbon source. Consumption of detected aromatic monomers and production of itaconic acid from depolymerized lignin containing either (e) $2 \mathrm{mM}$ or (f) $3 \mathrm{mM}$ supplemented $\mathrm{NH}_{4} \mathrm{Cl}$ in shake flask cultivations with strain JE3715. a-f Data are represented as the mean \pm standard deviation in three replicates, with the exception of $48 \mathrm{~h}$ time point in panel $\mathbf{f}$, which only contains two replicates and has no error bars. Source data are provided as a Source Data file.

isomer comprises $88 \%$ of aconitate at equilibrium and is a competitive inhibitor of the aconitase enzyme ${ }^{42}$-both features that could increase substrate accumulation and therefore increase flux to itaconic acid.

Taken together, we hypothesized that the trans-pathway would improve itaconic acid production relative to the cis-pathway by providing a thermodynamically favorable route to divert carbon flux from the TCA cycle. To test this, we integrated codonoptimized tad1 and adil genes under the control of the T7 promoter into strains JE3674 (icd ${ }^{\mathrm{GTG}}: i d h^{\mathrm{GTG}}$ ) and JE3681 (icd ${ }^{\text {TTG }}: i d h^{\text {TTG }}$ ) and assayed the resulting strains (JE3715 and JE3719, respectively) for itaconic acid production (Fig. 4d). As hypothesized, strains expressing the trans-pathway from $U$. maydis produced higher molar yields than equivalent strains expressing the cis-pathway (Fig. 4c, Table 1), and JE3719 produced the highest itaconic acid yield $(56 \% \mathrm{~mol} / \mathrm{mol}$ overall yield) from $p$-coumaric acid in this study, with a production phase yield of $116 \% \mathrm{~mol} / \mathrm{mol}$ which is $88 \%$ of the maximum theoretical yield of $1.33 \mathrm{~mol}$ itaconic acid/mol $p$-coumarate.

Production of itaconic acid from depolymerized lignin and other substrates. To test the viability of itaconic acid production from lignin, we assayed the ability of strain JE3715 to upgrade a depolymerized lignin stream produced from a lignocellulose deconstruction process ${ }^{3}$ to itaconic acid. Base-catalyzed depolymerization of corn stover lignin was performed as described previously ${ }^{3}$, and the resulting liquor was diluted with concentrated modified M9 salts containing either 2 or $3 \mathrm{mM} \mathrm{NH}_{4} \mathrm{Cl}$. The liquor provides $10.7 \mathrm{mM}(1.74 \mathrm{~g} / \mathrm{L}) p$-coumaric acid, $0.5 \mathrm{mM}$ $(0.09 \mathrm{~g} / \mathrm{L})$ ferulic acid, trace amounts of other monomeric carbon sources, and residual higher molecular weight lignin. JE3715, which contains the dynamically regulated trans-pathway and the GTG start codons for icd and idh, was chosen as the biocatalyst as its substantially higher growth rate and relatively similar yield enables much higher volumetric productivity relative to JE3717, and because JE3719 did not completely consume the provided substrate (Table 1). This strain was inoculated into shake flasks containing the two media variants and assayed for itaconic acid production. Production of itaconic acid leveled off at $48 \mathrm{~h}$ with titers between 1.40 and $1.43 \mathrm{~g} / \mathrm{L}$ (Fig. $4 \mathrm{e}-\mathrm{f}$ ). The high apparent yields $(99 \%$ overall molar yield and $0.79 \mathrm{~g}$ itaconic acid/g aromatic monomer) suggest that there may be other components in the lignin stream that lead to product and/or that the strain is breaking down and utilizing oligomeric lignin, as previously described ${ }^{43}$. Finally, to demonstrate the general utility of this 
organism and pathway, we demonstrated itaconic acid production from other potential waste stream feedstocks, including other aromatic compounds (benzoic acid, ferulic acid), sugars (glucose, xylose, arabinose), glycerol, and organic acids (acetic acid, succinic acid, octanoic acid) (Supplementary Table 4). With JE3715, the overall itaconic acid yield from ferulic acid $(51.6 \% \mathrm{~mol} / \mathrm{mol})$ was similar to $p$-coumaric acid in the same experiment $(56 \% \mathrm{~mol} /$ mol), and overall yield from benzoic acid reached $24 \%$ of the theoretical maximum yield without any optimization.

\section{Discussion}

Until recently, robust itaconic acid production was limited to sugar-utilizing fungi such as A. terreus and $U$. maydis, but in recent years, bacterial strains have been engineered to produce itaconic acid from glucose $e^{44,45}$, acetate ${ }^{46}$, and glycerol. ${ }^{47}$ However, all efforts to engineer heterologous itaconic acid production relied on expression of the cis-pathway (cadA) from A. terreus. ${ }^{31}$ In our work, the trans-pathway (adil/tad1) from U. maydis ${ }^{41}$ outperformed the cis-pathway, likely due to the production of a thermodynamically favorable intermediate. Accordingly, the trans-pathway may improve performance in other organisms. Among bacteria, the itaconic acid yield with $P$. putida, $0.44 \mathrm{~g} / \mathrm{g}$ from $p$-coumaric acid, compares well to an engineered $E$. coli that produced $0.5 \mathrm{~g}$ itaconic acid/g glucose. ${ }^{45}$ Furthermore, $1.43 \mathrm{~g} / \mathrm{L}$ itaconic acid was produced from a complex, depolymerized lignin stream, yielding $0.79 \mathrm{~g}$ itaconic acid $/ \mathrm{g}$ detectable aromatic monomers. Of note, the yield during the production phase is substantially higher than the overall yield in all cases, reaching nearly $1.2 \mathrm{~mol}$ itaconic acid/mol $p$-coumaric acid (the theoretical maximum yield is $1.33 \mathrm{~mol} / \mathrm{mol}$ ) using trans-pathway strains (Fig. 4c, Table 2). Therefore, advanced feeding strategies with extended production phases could improve yields even further. Finally, this work demonstrates itaconic acid production in an engineered bacterium in minimal salts medium without the use of replicating plasmids, antibiotic selections, or expensive inducer chemicals (e.g. IPTG), each of which will be critical for a commercial process.

Dynamic metabolic control combined with two-stage production is promising approach for biological chemical production ${ }^{30}$, and the biosensor developed here can serve as a master regulator for additional dynamic metabolic control tools such as CRISPRi $^{48}$, targeted proteolysis, and nested dynamic regulatory systems. Our nitrogen biosensor will be a valuable tool for future $P$. putida metabolic engineering because it is able to tune the amplitude of the nitrogen-starvation induced transcriptional response over an 89 -fold range, increasing it by up to 60 -fold over the original response. Despite its utility, to the best of our knowledge, $l_{s} Y$ has not previously been used for metabolic engineering. The dynamic response of this biosensor, already allowing 200-fold induction, has the potential to be further tuned by altering $l y s Y$ expression. Although not explicitly demonstrated here, one can modulate levels of sequentially utilized nitrogen sources such as $\mathrm{NH}_{4}$ and $\mathrm{NO}_{3}$ for auto-induction of gene expression in single-stage processes following depletion of $\mathrm{NH}_{4}$. Finally, nitrogen-responsive $\sigma^{54}$ promoters from $P$. putida similar to $\mathrm{P}_{\text {urt }}$ have been ported to E. coli ${ }^{36}$, suggesting this biosensor can work in other organisms expressing the activator NtrC.

In addition to lignin, recent work has demonstrated the potential for $P$. putida to valorize other biomass streams including thermochemical wastewater ${ }^{49}$ and lignocellulosic sugar hydrolysates ${ }^{50}$. Here, we demonstrated itaconic acid production from multiple substrates that could be found in potential commercial feedstocks, including biodiesel waste (glycerol), plant biomass (sugars, aromatics, acetic acid), and lipids (octanoic acid). Therefore, the technology developed here can serve as a universal platform for the two-phase production of a portfolio of chemicals from alternate carbon sources beyond lignin.

\section{Methods}

General culture conditions and media. The strains and plasmids used in this study are listed in Supplementary Table 5. Routine cultivation of Escherichia coli for plasmid construction and maintenance was performed at $37^{\circ} \mathrm{C}$ using $\mathrm{LB}$ (Miller) medium supplemented with $50 \mu \mathrm{g} / \mathrm{mL}$ kanamycin sulfate and $15 \mathrm{~g} / \mathrm{L}$ agar (for solid medium). All Pseudomonas putida cultures were incubated at $30^{\circ} \mathrm{C}$, with shaking at $250 \mathrm{rpm}$ for shake flask cultures with $p$-coumarate, shaking at $225 \mathrm{rpm}$ for BCDL medium shake flask cultures, and $548 \mathrm{rpm}$ with a $2 \mathrm{mM}$ orbital for cultures performed in a Neo2SM plate reader (BioTek). LB (Miller) was used for routine Pseudomonas putida strain maintenance, competent cell preparations, and starter cultures. For itaconate production assay starter cultures, the media was supplemented with $50 \mu \mathrm{g} / \mathrm{mL}$ kanamycin sulfate.

Modified M9 medium (M9*) with variable amounts of $\mathrm{NH}_{4} \mathrm{Cl}$ was utilized for shake flask experiments, growth rate assays, and fluorescent reporter assays ( $47.8 \mathrm{mM} \mathrm{Na}_{2} \mathrm{HPO}_{4}, 22 \mathrm{mM} \mathrm{KH}_{2} \mathrm{PO}_{4}, 8.6 \mathrm{mM} \mathrm{NaCl}, 1 \mathrm{mM} \mathrm{MgCl} 2,0.1 \mathrm{mM} \mathrm{CaCl}$, $18 \mu \mathrm{M} \mathrm{FeSO}_{4}, 1 \mathrm{x}$ MME trace minerals, $\mathrm{pH}$ adjusted to 7 with $\left.\mathrm{KOH}\right)$. In total, 1000x MME trace mineral stock solution contains per liter, $1 \mathrm{~mL}$ concentrated $\mathrm{HCl}, 0.5 \mathrm{~g}$ $\mathrm{Na}_{4}$ EDTA, $2 \mathrm{~g} \mathrm{FeCl}_{3}, 0.05 \mathrm{~g}$ each $\mathrm{H}_{3} \mathrm{BO}_{3}, \mathrm{ZnCl}_{2}, \mathrm{CuCl}_{2} \cdot 2 \mathrm{H}_{2} \mathrm{O}, \mathrm{MnCl}_{2} \cdot 4 \mathrm{H}_{2} \mathrm{O}$, $\left(\mathrm{NH}_{4}\right)_{2} \mathrm{MoO}_{4}, \mathrm{CoCl}_{2} \cdot 6 \mathrm{H}_{2} \mathrm{O}, \mathrm{NiCl}_{2} \cdot 6 \mathrm{H}_{2} \mathrm{O}$. Unless otherwise noted, all $\mathrm{M}{ }^{*}$ medium was supplemented with $20 \mathrm{mM}$ p-coumarate (neutralized with $\mathrm{NaOH}$ ) as a sole carbon source. MME medium (containing $9.1 \mathrm{mM} \mathrm{K}_{2} \mathrm{HPO}_{4}, 20 \mathrm{mM}$ MOPS, $4.3 \mathrm{mM} \mathrm{NaCl}, 0.41 \mathrm{mM} \mathrm{MgSO}_{4}, 68 \mu \mathrm{M} \mathrm{CaCl}_{2}, 1 \mathrm{x}$ MME trace minerals, $\mathrm{pH}$ adjusted to 7.0 with $\mathrm{KOH}$ ) supplemented with $20 \mathrm{mM}$ glucose and either $20 \mathrm{mM}$ $\mathrm{NH}_{4} \mathrm{Cl}$ or $20 \mathrm{mM} \mathrm{NaNO}$ was utilized for transcriptomics experiments.

Production of BCDL and depolymerized lignin media preparation. The preparation of BCDL has been reported before. ${ }^{32}$ Specifically, dry solid material remaining from the enzymatic hydrolysis of pretreated corn stover (which follows the biorefinery process designed at NREL $\left.{ }^{51}\right)$ was added as $10 \%(\mathrm{w} / \mathrm{v})$ solids to a $2 \%$ $\mathrm{NaOH}$ solution and loaded into $200 \mathrm{~mL}$ stainless steel reactors. The reaction was carried out at $120^{\circ} \mathrm{C}$ for $30 \mathrm{~min}$. The sterile and solubilized material was neutralized with $4 \mathrm{~N} \mathrm{H}_{2} \mathrm{SO}_{4}$ and centrifuged at $10,875 \times g$ for $20 \mathrm{~min}$ in aseptic conditions. Then, the supernatant $(90 \% \mathrm{v} / \mathrm{v})$ was mixed with $10 \mathrm{x} \mathrm{M}{ }^{*}$ salts (without any nitrogen source) and $\mathrm{NH}_{4} \mathrm{Cl}$ to generate $\mathrm{M} 9{ }^{*}-\mathrm{BCDL}$ medium supplemented with either $2 \mathrm{mM}$ or $3 \mathrm{mM} \mathrm{NH}_{4} \mathrm{Cl}$.

Plasmid and Pseudomonas strain construction. Phusion ${ }^{\circledR}$ HF Polymerase (Thermo Scientific) and primers synthesized by Eurofins Genomics were used in all PCR amplifications for plasmid construction. OneTaq ${ }^{\circledR}$ (New England Biolabs NEB) was used for colony PCR. Plasmids were constructed by Gibson Assembly using NEBuilder ${ }^{\circledast}$ HiFi DNA Assembly Master Mix (NEB) or ligation using T4 DNA ligase (NEB). Plasmids were transformed into either competent NEB 5-alpha F'Iq (NEB), Epi400 (Lucigen), or QP15 (Epi400 mated with NEB 5-alpha F'I to transfer the mini F' plasmid to Epi400). Standard chemically competent Escherichia coli transformation protocols were used to construct plasmid host strains. Transformants were selected on LB (Miller) agar plates containing $50 \mu \mathrm{g} / \mathrm{mL}$ kanamycin sulfate for selection and incubated at $37^{\circ} \mathrm{C}$. Template DNA was either synthesized by IDT or isolated from E. coli or P. putida KT2440 using Zymo Quick gDNA miniprep kit (Zymo Research). Zymoclean Gel DNA recovery kit (Zymo Research) was used for all DNA gel purifications. Plasmid DNA was purified from E. coli using GeneJet plasmid miniprep kit (ThermoScientific) or ZymoPURE plasmid midiprep kit (Zymo Research). Sequences of all plasmids were confirmed using Sanger sequencing performed by Eurofins Genomics. Plasmids used in this work are listed in Supplementary Table 5, and details regarding plasmid construction and sequences are below. All DNA oligos used in this work can be found in Supplementary Table 6.

P. putida JE90, a derivative of $P$. putida KT2440 where the restriction endonuclease $h s d R$ has been replaced with the Bxb1-phage integrase and respective attB sequence ${ }^{32}$, was used as a parent for all $P$. putida strains used in this study (Supplementary Table 5). All genome modifications were performed using either the homologous recombination-based pK18mobsacB kanamycin resistance/sucrose sensitivity selection/counter-selection system ${ }^{52,53}$ or with the Bxb1-phage integrase system $^{32}$ with minor modifications to competent cell preparation procedures. Electrocompetent Pseudomonas putida were prepared as follows. Strains were inoculated into LB broth and incubated at $30^{\circ} \mathrm{C}, 250 \mathrm{rpm}$ until they reached stationary phase-typically $\sim 16 \mathrm{~h}$. Cells were centrifuged at $3000 \times g$ for $20 \mathrm{~min}$ at room temperature and following decanting of supernatant they were washed by resuspended in $1 / 2$ the original culture's volume of room temperature $10 \%$ glycerol. Resuspended cells were centrifuged, decanted and resuspended in $10 \%$ glycerol two additional times. Following the final centrifugation, cells were resuspended in $1 / 50^{\text {th }}$ the original culture's volume of $10 \%$ glycerol. Cells were either used immediately or stored at $-80^{\circ} \mathrm{C}$. For transformation, $5 \mu \mathrm{L}(200 \mathrm{ng}-2 \mu \mathrm{g})$ of plasmid DNA was added to $50 \mu \mathrm{L}$ of the electrocompetent cells, transferred to a $0.1 \mathrm{~cm}$ electroporation cuvette, and and electroporated at $1.6 \mathrm{kV}, 25 \mathrm{uF}, 200 \Omega$. Electroporated cells were resuspended following addition of $950 \mu \mathrm{L}$ SOC medium, transferred to a $1.5 \mathrm{~mL}$ microcentrifuge tube, and incubated with $250 \mathrm{rpm}$ shaking 
for $1 \mathrm{~h}$ at $30^{\circ} \mathrm{C}$. Dilutions of the recovery cultures were plated onto LB agar medium supplemented with $50 \mu \mathrm{g} / \mathrm{mL}$ kanamycin sulfate. For plasmids integrated using Bxb1 integrase transformants were screened by colony PCR to verify plasmid insertion into the genome. For strains constructed using homologous

recombination, transformants were streaked for single colony isolation on LB agar medium supplemented with $50 \mu \mathrm{g} / \mathrm{mL}$ kanamycin sulfate and incubated overnight at $30^{\circ} \mathrm{C}$ to select against residual wild-type cells that are insensitive to sucrose. For sucrose counter-selection, restreaked transformants were streaked for single colonies on YT $+25 \%$ sucrose plates $(10 \mathrm{~g} / \mathrm{L}$ yeast extract, $20 \mathrm{~g} / \mathrm{L}$ tryptone, $250 \mathrm{~g} / \mathrm{L}$ sucrose, and $18 \mathrm{~g} / \mathrm{L}$ agar), and incubated at $30^{\circ} \mathrm{C}$ overnight. Colonies were streaked a second time on YT $+25 \%$ sucrose, and screened by colony PCR for desired mutations. Gene deletions and replacements were performed by homologous recombination, while integration of reporter and itaconate production pathway cassettes was performed with the Bxb1-phage integrase system. Primers used for screening $P$. putida strains for $p h a C_{1} Z C_{2}$ deletion, ampC::T7_RNAP replacements, and icd/idh start codon swaps can be found in Supplementary Table 6. Integration of pJE990-derivatives using the phage integrase system was confirmed by colony PCR using oligos oJE66 \& oJE536.

Plasmid construction details. Annotated sequences of all plasmids can be found in Supplementary Data 3. All enzymes used for plasmid construction were purchased from NEB. Supplementary Table 6 contains the sequence of all oligos listed below.

For construction of pJE473, homology arms to target deletion of pha $C_{1} Z C_{2}$ (PP_5003-5005) were amplified by PCR from wild-type $P$. putida genomic DNA using primer combinations oJE331/332 and oJE333/334, assembled into gel purified EcoRI/HindIII-linearized pJE382, and transformed into NEB 5-alpha F'IQ. Resulting E. coli colonies were screened by colony PCR for the presence of homology arms using primers oJE255/256. Candidates for pJE473 were purified from E. coli and sequenced using primers oJE255/256.

For construction of pJE387, homology arms to target deletion of $a m p C$ (PP_2876) were amplified by PCR from wild-type P. putida genomic DNA using primer combinations oJE89/90 and oJE91/92, assembled into gel purified EcoRI/ HindIII-linearized pK18mobsacB, and transformed into NEB 5-alpha F'IQ. Resulting E. coli colonies were screened by colony for the presence of homology arms using primers oJE255/256. Candidates for pJE387 were purified from E. coli and sequenced using primers oJE255/256.

For construction of pJE382, we utilized QuikChange PCR mutagenesis with oligos oJE69/70 to amplify a version of pK18mobsacB with the lac promoter that drives expression of the lacZalpha fragment deleted. Following amplification, the PCR reaction was digested with $20 \mathrm{U}$ of DpnI (NEB) for $1 \mathrm{~h}$ at $37^{\circ} \mathrm{C}$ to reduce parental plasmid background. The purpose of this plasmid was to reduce likelihood of recombination with lac/tac-based promoters in $\mathrm{pK} 18 \mathrm{mobsacB}$ with similar promoters in our synthetic constructs, and to reduce potential toxicity from unintended transcription of homology arms/expression cassettes in E. coli. The DpnI-digested PCR product was transformed into NEB 5-alpha F' $I^{Q}$. Resulting $E$. coli colonies were screened by colony for the presence of the lac promoter using primers oJE71/72. Candidates for pJE382 were purified from E. coli and lac promoter deletion verified by sequencing with primer oJE72.

For construction of pJE1031, homology arms for the deletion of $a m p C$ (PP_2876) were amplified from pJE387 using primer combination oJE92/608, assembled into gel purified EcoRI/HindIII-linearized pJE382, and transformed into NEB 5-alpha F'IQ. Resulting E. coli colonies were screened by colony PCR for the presence of homology arms using primers oJE255/256. Candidates for pJE1031 were purified from E. coli and sequenced using primers oJE255/256.

For construction of pJE1032, pJE1033, pJE1037, and pJE1039 promoter sequences containing $200-300$ bp upstream of PP_2685, PP_2688, urtA (PP_4841), and $g \ln K$ (PP_5234), respectively, were amplified from P. putida (Supplementary Table 6 for oligos) and assembled with T7 RNAP and a synthetic terminator sequence. The T7 RNAP polymerase and a downstream terminator was amplified from BL21(DE3) pLysS (Promega) genomic DNA using oligos oJE625/ 626. A double terminator sequence for insulation of the construct was amplified from the T7 dbl term gBlock using oJE627/628. Parts were assembled into BamHI/XbaI-linearized pJE1031, and transformed into NEB 5-alpha F'Q. Resulting E. coli colonies were screened by colony PCR using primers oJE177/178. Candidates for the plasmids were purified from E. coli and sequenced using oJE177/178/631/632/633.

For construction of the reporter plasmids we annealed oligos containing desired promoter sequences and ligated the promoters into a promoterless $\mathrm{mNeonGreen}$ reporter plasmid, pJE990. Plasmid pJE990 was linearized with BbsI. Promoter oligos pairs were phosphorylated with PNK (NEB) in T4 DNA ligase buffer, annealed by heating to $95^{\circ} \mathrm{C}$ and cooling at $1{ }^{\circ} \mathrm{C} /$ minute to room temperature. Annealed oligo sets oJE634/635, oJE97/98/133/134, oJE826/827, oJE828/829, oJE830/831, and oJE832/833 were ligated to BbsI-linearized pJE990 to construct plasmids pJE1040, pJE1045, pJE1118, pJE1119, pJE1120, and pJE1121 respectively. Ligated DNA was transformed into NEB 5-alpha F' ${ }^{\mathrm{Q}}$. Plasmids were isolated from transformant colonies and confirmed by sequencing with oJE535. For construction of mKate2 variant plasmids, mKate2 was amplified from the mKate2 gBlock using oligos oJE1724/1725 and digested with NdeI/XbaI. Plasmids pJE1045, pJE1040 and pJE1118-1121 were digested with NdeI/XbaI and ligated with NdeI/XbaI digested
mKate2 gBlock to generate plasmids pGW55, pJE1454-1458. Ligations were transformed into NEB 5-alpha F' $\mathrm{I}^{\mathrm{Q}}$, and candidates confirmed by sequencing of isolated plasmid DNA using oligos oJE535/536.

For construction of pJE1180 we amplified the cat and lys $S$ genes from pLysS (Promega) as two parts with primers designed to introduce the lys $Y$ mutation, assembled the resulting parts into SpeI-linearized pJE1040. Primers oJE817/818 and oJE819/820 were used to amplify the two parts. The resulting lys Y/cat fragment was digested with SpeI and ligated into XbaI-linearized pJE1037, generating plasmid pJE1180.

For construction of pJE1380, codon-optimized cadA from Aspergillus terreus was assembled into NdeI/XbaI-linearized pJE1040-replacing mNeonGreen. The cadA gene was synthesized as gBlocks cadA_gBlock_1 \& cadA_gBlock_2. gBlocks 1 \& 2 were amplified using oligos oJE1408/1409 and oJE1410/1411, respectively. The assembly was transformed into NEB 5-alpha $\mathrm{F}^{\prime} \mathrm{I}^{\mathrm{Q}}$, and transformants were screened using oJE535/536. Plasmid DNA was isolated from PCR positive candidates and sequenced using oJE535/536/1412.

For pJE1390, the cadA gene from pJE1380 was excised using NdeI/XbaI, and ligated into NdeI/XbaI linearized pJE1045. The ligation was transformed into QP15, and transformants were screened by colony PCR using oligos oJE535/536. The assembly was transformed into NEB 5-alpha F' ${ }^{\mathrm{Q}}$, and transformants were screened using oJE535/536. Plasmid DNA was isolated from PCR positive candidates and sequenced using oJE535/536/1412.

For pJE1443, codon-optimized tad1 and adil genes from Ustilago maydis were assembled into AflIII/XbaI-linearized pJE1040 - replacing mNeonGreen and its RBS sequence. The tad 1 and adil genes were synthesized as gBlocks tad 1 and adil, which were amplified using primer combinations oJE1554/1547 and oJE1555/1548, respectively. The assembly was transformed into NEB 5-alpha F' $\mathrm{IQ}$, and transformants were screened using oJE535/536. Plasmid DNA was isolated from PCR positive candidates and sequenced using oJE535/536/1559/1560/1561.

For the construction of the icd / idh start codon swap plasmids pJE1444 and pJE1445, we assembled several PCR reactions containing homology arms for targeting, and mutations in the start codons (and RBS neutral mutations in the region between core RBS and start codon) of icd \& idh. The homology arms for targeting insertion of the two plasmids into the $i c d / i d h$ locus were amplified using primer pairs oJE1564/1565 and oJE1568/1569 for both plasmids. The central fragment contained between the two homology arms, containing the various mutations, was amplified using oligos oJE1566/1567 for pJE1444 and oligos oJE1570/1571 for pJE1445. The parts were assembled into EcoRI/HindIIIlinearized pJE382, transformed into NEB 5-alpha $\mathrm{FJ}^{\mathrm{Q}}$, and transformants were screened using oJE255/256. Plasmid DNA was isolated from PCR positive candidates and sequenced using oJE255/256/1572/1573.

For construction of pJE365, homology arms to target deletion of $g c d$ (PP_1444) were amplified by PCR from wild-type $P$. putida genomic DNA, assembled into gel purified XbaI/HindIII-linearized $\mathrm{pK} 18 \mathrm{mobsacB}$, and transformed into NEB 5alpha $\mathrm{F}^{\mathrm{Q}} \mathrm{Q}$. Resulting E. coli colonies were screened by colony for the presence of homology arms using primers oJE255/256. Candidates for pJE365 were purified from E. coli and sequenced using primers oJE255/256.

For construction of plasmid pJE1345, we had IDT synthesize gBlocks containing a $P$. putida codon-optimized $\operatorname{araA}_{2}-\mathrm{araE}_{2}$ oxidative L-arabinose catabolic pathway cassette from Burkholderia ambifaria AMMD and a P. putida codon-optimized E. coli $\operatorname{araE}_{1} \mathrm{~L}$-arabinose: $\mathrm{H}^{+}$symporter cassette. The gBlocks were assembled in between $\Delta g c d$ homology arms in pJE365 backbone and transformed into NEB 5-alpha F'IQ. Resulting E. coli colonies were screened by restriction digest for the presence of the properly assembled $B$. ambifaria araCDABE cassette. Candidates for pJE1345 were purified from E. coli and confirmed by Sanger sequencing. See plasmid map for sequence details.

For construction of pJE1479, we had IDT or GenScript synthesize portions of a cassette that contains a P. putida codon-optimized E. coli xylE xylose: $\mathrm{H}^{+}$symporter expression cassette that is divergent from a $P$. putida codon-optimized Burkholderia xenovorans xylCDBX oxidative xylose catabolic pathway expression cassette. These cassettes were assembled using Gibson Assembly with homology arms to target insertion downstream of $f p v A$ in the Pseudomonas putida KT2440 genome. See plasmid map for sequence details.

Plasmid pJE1045 was PCR amplified using primers pJE1045-ATGGTCf / pJE1045-ATGGTCr, pJE1045-GTGGTCf / pJE1045-GTGGTCr, and pJE1045 TTGGTCf / pJE1045-TTGGTCr to produce plasmids pJE1045-ATGGTC, pJE1045-GTGGTC, and pJE1045-ATGGTC, respectively by PCR mutagenesis. The resulting PCR products were digested with DpnI, transformed into NEB 5-alpha F'T $\mathrm{Q}$. Plasmids from resulting colonies were screened by Sanger sequencing.

Growth rate analysis. LB medium was inoculated from glycerol stocks and incubated overnight at $30^{\circ} \mathrm{C}, 250 \mathrm{rpm}$ for precultures. Cultures were washed twice by centrifugation $(\sim 4000 \times g$ for $10 \mathrm{~min})$ and resuspension in equal volumes of $1 \mathrm{x}$ M9 salts lacking $\mathrm{NH}_{4} \mathrm{Cl}$ to remove residual $\mathrm{LB}$ medium and resuspended in $1 / 3$ volume $1 \mathrm{x} \mathrm{M} 9$ salts. Optical density $\left(\mathrm{OD}_{600}\right)$ of resulting suspensions was measured using a $1 \mathrm{~cm}$ pathlength cuvette. Growth assays were performed with $600 \mathrm{uL} \mathrm{M9*}$ medium supplemented with $20 \mathrm{mM} p$-coumarate and $20 \mathrm{mM} \mathrm{NH}_{4} \mathrm{Cl}$ in clear 48 well microtiter plates with an optically clear lid (Greiner Bio-One). All cultures were inoculated with washed cultures to an $\mathrm{OD}_{600}$ equivalent to 0.03 in a $1 \mathrm{~cm}$ pathlength cuvette. Plates were incubated at $30^{\circ} \mathrm{C}$, fast shaking in an Epoch2 plate 
reader (Bio-Tek) using Gen5 software (V3.0), with $\mathrm{OD}_{600}$ readings taken every 10 minutes. Exponential growth rates were determined using the CurveFitter software (Version 1; http://www.evolvedmicrobe.com/CurveFitter/) with data points in early mid-log phase. All growth rates were calculated from 3 replicate experiments. Standard deviations are two-sided.

Fluorescent reporter assays. Strains were revived from glycerol stocks in $5 \mathrm{~mL}$ LB with overnight incubation at $30^{\circ} \mathrm{C}, 250 \mathrm{rpm} .5 \mathrm{~mL}$ starter cultures in $\mathrm{M} 9^{*}+$ $20 \mathrm{mM}$ glucose $+10 \mathrm{mM} \mathrm{NH}_{4} \mathrm{Cl}$ were inoculated with $1 \%$ of the recovery culture and similarly incubated. Coupled growth and fluorescence assays were performed with a Neo2SM (Bio-Tek) plate reader using $200 \mu \mathrm{L} /$ well of $\mathrm{M}^{*}+20 \mathrm{mM}$ pcoumarate $+2 \mathrm{mM}$ (limiting) or 20 (replete) $\mathrm{mM} \mathrm{NH}_{4} \mathrm{Cl}$ in black-walled, $\mu$ Clear ${ }^{\otimes}$ flat-bottom, 96-well plates (Greiner Bio-One) with an optically clear lid. Plate cultures were inoculated with $0.5 \%$ inoculum from starter cultures, and incubated overnight at $30^{\circ} \mathrm{C}$, fast shaking with $\mathrm{OD}_{600}$ and fluorescence $\left(\mathrm{F}_{510,530}\right.$ for $\mathrm{mNeonGreen}$ and $\mathrm{F}_{588,633}$ for mKate2) measured every 10 minutes. Reporter expression per cell was estimated by dividing relative fluorescence units (RFU) by $\mathrm{OD}_{600}$ (as a proxy for cell number) for each time point and averaging those values for time points occurring during either exponential growth or stationary phase. Background absorbance and fluorescence readings from wells containing media blanks were averaged and subtracted from sample readings prior to analysis. Exponential phase was defined as time points where $\mathrm{OD}_{600}$ was between 0.039 and the $\mathrm{OD}_{600}$ curve inflection point, typically $\mathrm{OD}_{600} \sim 0.2$ (nitrogen limited) or $\sim 0.6$ (nitrogen replete). Stationary phase was defined as time points starting $2 \mathrm{~h}$ following end of exponential phase. Standard deviations are two-sided.

Shake flask experiments for itaconate production. Starter cultures were prepared as described for growth rate assays with the exception that $50 \mu \mathrm{g} / \mathrm{mL}$ kanamycin sulfate was added to the medium. Starter cultures were inoculated to a final $\mathrm{OD}_{600}$ of 0.1 ( $p$-coumarate cultures) or 0.2 (BCDL cultures) into $25 \mathrm{~mL}$ of M9*-coumarate or M9*-BCDL medium, supplemented with either $2 \mathrm{mM} \mathrm{NH}_{4} \mathrm{Cl}$ (all p-coumarate cultures) or $3 \mathrm{mM}$ (BCDL cultures), in a $125 \mathrm{~mL}$ Erlenmeyer flask and incubated at $30^{\circ} \mathrm{C}, 250 \mathrm{rpm}$. Cultures were sampled periodically to measure growth by $\mathrm{OD}_{600}$, and analyte concentrations by high performance liquid chromatography (HPLC).

Analytical techniques. For shake flask experiments with $\mathrm{M} 9{ }^{*}$-p-coumarate, optical density at $600 \mathrm{~nm}\left(\mathrm{OD}_{600}\right)$ was measured directly from cultures using a spectrophotometer (Amersham, UltroSpec10) blanked with M9*-coumarate. HPLC analysis for $p$-coumarate and organic acid detection was performed by injecting $20 \mu \mathrm{L}$ of $0.2 \mu \mathrm{m}$ filtered culture supernatant onto a Waters 1515 series system equipped with a Rezex RFQ-Fast Acid H + (8\%) column (Phenomenex) and a Micro-Guard Cation $\mathrm{H}^{+}$cartridge (Bio-Rad). Samples were run with column at $60^{\circ} \mathrm{C}$ using a mobile phase of $0.01 \mathrm{~N}$ sulfuric acid at a flow rate of $0.6 \mathrm{~mL} / \mathrm{min}$, with a refractive index detector and UV/Vis detector measuring $A_{230} \& A_{280}$ for analyte detection. Analytes were identified and quantified by comparing retention times and spectra with pure standards.

For shake flask experiments with M9*-BCDL, optical density at $600 \mathrm{~nm}$ $\left(\mathrm{OD}_{600}\right)$ was measured with a Nanodrop (ThermoFisher Scientific) after diluting samples sixfold. Uninoculated M9*-BCDL medium was used as a blank to subtract signal coming from components in the medium.

Itaconic acid quantitation in M9*-BCDL. Prior the analysis, a $0.1 \mathrm{~mL}$ aliquot was taken from each sample and $0.9 \mathrm{~mL}$ of water were added to make a 10x dilution. Then, $34 \mu \mathrm{L}$ of $72 \%$ sulfuric acid were added to each diluted sample to decrease the $\mathrm{pH}$ below 2.0 and precipitate acid insoluble lignin. Samples were centrifuged, and the supernatant was filtered through a $0.2 \mu \mathrm{M}$ filter pore size. Itaconic acid quantification was performed on an Agilent 1100 series HPLC system, with a diode array detector (DAD) at $210 \mathrm{~nm}$ (Agilent Technologies). Analysis was performed by injecting $6 \mu \mathrm{L}$ of filtered culture supernatant onto a Phenomenex Rezex ${ }^{\text {tw }}$ RFQ-Fast Acid $\mathrm{H}^{+}(8 \%)$ column with a cation $\mathrm{H}^{+}$guard cartridge (Bio-Rad Laboratories) at $85^{\circ} \mathrm{C}$ using a mobile phase of $5 \mathrm{mM}$ sulfuric acid at a flow rate of $1.0 \mathrm{~mL} / \mathrm{min}$

Aromatic compounds quantitation in M9*-BCDL. Metabolite analysis in $\mathrm{BCD}$ was performed on an Agilent 1200 LC system (Agilent Technologies) equipped with a DAD. Each sample and standard was injected at a volume of $10 \mu \mathrm{L}$ onto a Phenomenex Luna C18(2) column $5 \mu \mathrm{m}, 4.6 \times 150 \mathrm{~mm}$ column (Phenomenex). The column temperature was maintained at $30^{\circ} \mathrm{C}$ and the buffers used to separate the analytes of interest were (A) $0.05 \%$ acetic acid in water and (B) $0.05 \%$ acetic acid in acetonitrile. The chromatographic separation was carried out using a gradient of: initially starting at $1 \%$ B going to $50 \%$ B at 35 min before immediately switching to $99 \%$ B at $35.1 \mathrm{~min}$, before equilibrium for a total run time of $47 \mathrm{~min}$. The flow rate of the mobile phases was held constant at $0.6 \mathrm{~mL} / \mathrm{min}$. The same standards used in the BCDL experiments were also used to construct calibration curves, but between the ranges of 5-200 $\mu \mathrm{g} / \mathrm{L}$. Three separate wavelengths from the $\mathrm{DAD}$ were used to identify and quantitate the analytes of interest. A wavelength of $210 \mathrm{~nm}$ and $225 \mathrm{~nm}$ was used for the analytes vanillic acid and 4-hydroxybenzoic acid. A wavelength of $325 \mathrm{~nm}$ was used for the analytes $p$-coumaric acid, and ferulic acid. A minimum of five calibration levels was used with an $\mathrm{r}^{2}$ coefficient of 0.995 or better for each analyte. Standard deviations are two-sided.
Transcriptional profiling of $\boldsymbol{P}$. putida. For the determination of $\mathrm{NO}_{3}$ induced promoters, we utilized strain JE1657, an engineered P. putida strain containing a Bxb1 phage integrase system for rapid genomic integration of $\mathrm{DNA}^{32}$, and a $\mathrm{P}_{\mathrm{T} 7}$ : mNeonGreen reporter cassette. JE1657 was cultured at $30 \mathrm{C}$ in $50 \mathrm{~mL}$ MME mineral medium in a $250 \mathrm{~mL}$ Erlenmeyer shake flask at $30^{\circ} \mathrm{C}, 250 \mathrm{rpm}$ shaking and harvested mid-log $\left(\mathrm{OD}_{600}=\sim 0.2\right)$ by centrifugation $\left(\sim 16,000 \times g, 2 \mathrm{~min}, 4^{\circ} \mathrm{C}\right)$. Supernatants were quickly decanted, and cell pellets were frozen rapidly in liquid nitrogen prior to storage at $-80^{\circ} \mathrm{C}$ for storage prior to RNA isolation. Four samples were prepared for each condition. For characterization of biosensor performance, strain JE2212 was cultured under identical conditions.

Cell pellets, were resuspended in TRIzol (ThermoFisher-Invitrogen, Waltham, MA USA) and processed according to the manufactures protocol for TRIzol reagent. In general, TRIzol was added to cell pellets and mixed by vortex and pipetting. Chloroform was then added and mixed and samples were centrifuged. After centrifugation the aqueous layer was removed and mixed $1: 1$ with $80 \%$ ethanol. The samples were then purified on a RNeasy column (Qiagen Hilden, Germany) following the manufactures protocol and the on-column DNase digestion. RNA was eluted off the column in $35 \mu \mathrm{L}$ RNAse free $\mathrm{H}_{2} 0$ (Qiagen, Hilden, Germany). RNA concentration was quantified using a Nanodrop 1000 instrument (ThermoScientific, Waltham, MA) and RNA quality was verified by obtaining RNA Integrity Numbers (RIN) using an RNA 6000 Nanochip on an Agilent 2100 Bioanalyzer (Agilent Technologies, Santa Clara, CA).

Ribosomal RNA was depleted from total RNA samples using a RiboZero rRNA Removal Kit (Epicentre-Illumina Inc. San Diego, CA) according to manufacturer's instructions. The depleted sample was purified on a RNA Clean \& Concentrator-5 (Zymo Research, Irvine, CA, USA) following the manufacturer's protocol, and then the depleted material was quantified using a Nanodrop 1000 and visualized on an Agilent 2100 Bioanalyzer instrument with a RNA 6000 Nanochip (Agilent Technologies, Santa Clara, CA). RNA depleted of ribosomal RNA was used as input material to synthesize cDNA libraries using a ScriptSeq v2 RNA-Seq Library Preparation Kit (Illumina-Epicentre, San Diego, CA, USA) according to manufacturer's instructions and TruSeq compatible barcodes. Pooled barcoded libraries were sequenced in one direction for 50 bases (SE50) on an Illumina HiSeq2500 using v4 chemistry (Illumina Inc. San Diego, CA) and de-multiplexed as a sequencing service provided by The Genomic Services Lab at Hudson Alpha Institute for Biotechnology (HudsonAlpha, Huntsville, AL).

Differential gene expression analysis. After Illumina sequencing, RNA-seq reads were mapped to modified versions of the P. putida KT2440 reference genome (NC_002947) containing the mutations found in JE1657 and JE2212 using the Geneious for RNA-seq mapping workflow. Read count per annotated gene was calculated for each treatment and replicate, as well as fragment per kilobase million (FPKM), a common normalization technique. We then exported gene locus tags and raw read counts into tab-delimited files, one for each replicate. To calculate differential gene expression, we used the R package DESeq2 ${ }^{54}$ (version 1.10.0), which calculates log-fold change in expression and allows comparison between treatments using several replicates. We had three (JE2212 assay) or four (JE1657 assay) replicates per treatment, for a total of six or eight inputs per experiment. Differential expression data can be found in Supplementary Data 1 (JE2212) and Supplementary Data 2 (JE1657).

Statement on measurements. For all data points in this manuscript, measurements were taken from distinct samples.

Reporting summary. Further information on research design is available in the Nature Research Reporting Summary linked to this article.

\section{Data availability}

Data supporting the findings of this work are available within the paper and its Supplementary Information files. A reporting summary for this Article is available as a Supplementary Information file. The datasets, plasmids, and microorganisms generated and analyzed during the current study are available from the corresponding author upon request. NGS expression data generated in this study were deposited in the Gene Expression Omnibus (GEO) under accession number GSE147420. Source data are provided with this paper.

Received: 15 December 2020; Accepted: 17 March 2021; Published online: 15 April 2021

\section{References}

1. Davis, R. et al. Process design and economics for the conversion of lignocellulosic biomass to hydrocarbons: dilute-acid and enzymatic deconstruction of biomass to sugars and biological conversion of sugars to hydrocarbons. Technical Report NREL/TP-5100-60223, National Renewable Energy Laboratory, Golden, CO, pp. 88-101 (2013). 
2. Vardon, D. R. et al. Adipic acid production from lignin. Energy Environ. Sci. 8, 617-628 (2015).

3. Rodriguez, A. et al. Base-Catalyzed Depolymerization of Solid Lignin-Rich Streams Enables Microbial Conversion. Acs Sustain Chem. Eng. 5, 8171-8180 (2017).

4. $\mathrm{Wu}, \mathrm{W}$. H. et al. Lignin valorization: two hybrid biochemical routes for the conversion of polymeric lignin into value-added chemicals. Sci. Rep. 7 (2017).

5. Kohlstedt, M. et al. From lignin to nylon: Cascaded chemical and biochemical conversion using metabolically engineered Pseudomonas putida. Metab. Eng. 47, 279-293 (2018)

6. Barton, N. et al. Enabling the valorization of guaiacol-based lignin: Integrated chemical and biochemical production of cis,cis-muconic acid using metabolically engineered Amycolatopsis sp ATCC 39116. Metab. Eng. 45, 200-210 (2018).

7. Johnson, C. W. et al. Innovative chemicals and materials from bacterial aromatic catabolic pathways. Joule 3, 1523-1537 (2019).

8. Mycroft, Z., Gomis, M., Mines, P., Law, P. \& Bugg, T. D. H. Biocatalytic conversion of lignin to aromatic dicarboxylic acids in Rhodococcus josti RHA1 by re-routing aromatic degradation pathways. Green. Chem. 17, 4974-4979 (2015).

9. Linger, J. G. et al. Lignin valorization through integrated biological funneling and chemical catalysis. P Natl Acad. Sci. USA 111, 12013-12018 (2014).

10. Zhao, C. et al. Synergistic enzymatic and microbial lignin conversion. Green. Chem. 18, 1306-1312 (2016).

11. Kosa, M. \& Ragauskas, A. J. Bioconversion of lignin model compounds with oleaginous Rhodococci. Appl Microbiol Biotechnol. 93, 891-900 (2012).

12. Langholtz, M. H., Stokes, B. J. \& Eaton, L. M. Medium: ED; Size: 448 (EERE Publication and Product Library, 2016).

13. Cunha da Cruz, J., Machado de Castro, A. \& Camporese Servulo, E. F. World market and biotechnological production of itaconic acid. 3 Biotech 8, 138 (2018).

14. Klement, T. \& Buchs, J. Itaconic acid - A biotechnological process in change. Bioresour. Technol. 135, 422-431 (2013).

15. Kuenz, A. \& Krull, S. Biotechnological production of itaconic acid-things you have to know. Appl Microbiol Biotechnol (2018).

16. Werpy, T. et al. (Department of Energy Washington DC, 2004).

17. Nieder-Heitmann, M., Haigh, K. F. \& Gorgens, J. F. Process design and economic analysis of a biorefinery co-producing itaconic acid and electricity from sugarcane bagasse and trash lignocelluloses. Bioresour. Technol. 262, 159-168 (2018).

18. Horlamus, F. et al. Potential of biotechnological conversion of lignocellulose hydrolyzates by Pseudomonas putida KT2440 as a model organism for a biobased economy. Gcb Bioenergy 11, 1421-1434 (2019).

19. Nike, P. I., Chavarria, M., Danchin, A. \& de Lorenzo, V. From dirt to industrial applications: Pseudomonas putida as a Synthetic Biology chassis for hosting harsh biochemical reactions. Curr. Opin. Chem. Biol. 34, 20-29 (2016).

20. Ebert, B. E., Kurth, F., Grund, M., Blank, L. M. \& Schmid, A. Response of Pseudomonas putida KT2440 to increased NADH and ATP demand. Appl Environ. Microbiol 77, 6597-6605 (2011).

21. Ramos, J. L. et al. Mechanisms of solvent resistance mediated by interplay of cellular factors in Pseudomonas putida. FEMS Microbiol Rev. 39, 555-566 (2015).

22. Kieboom, J., Dennis, J. J., de Bont, J. A. \& Zylstra, G. J. Identification and molecular characterization of an efflux pump involved in Pseudomonas putida S12 solvent tolerance. J. Biol. Chem. 273, 85-91 (1998).

23. Fernandez, M. et al. Microbial responses to xenobiotic compounds. Identification of genes that allow Pseudomonas putida KT2440 to cope with 2,4,6-trinitrotoluene. Micro. Biotechnol. 2, 287-294 (2009).

24. Inoue, A. \& Horikoshi, K. A pseudomonas thrives in high-concentrations of toluene. Nature 338, 264-266 (1989).

25. Jimenez, J. I., Minambres, B., Garcia, J. L. \& Diaz, E. Genomic analysis of the aromatic catabolic pathways from Pseudomonas putida KT2440. Environ. Microbiol 4, 824-841 (2002).

26. Salvachua, D. et al. Metabolic engineering of Pseudomonas putida for increased polyhydroxyalkanoate production from lignin. Micro. Biotechnol. 13, 290-298 (2020).

27. Salvachua, D. et al. Bioprocess development for muconic acid production from aromatic compounds and lignin. Green. Chem. 20, 5007-5019 (2018).

28. McFadden, B. A. \& Purohit, S. Itaconate, an isocitrate lyase-directed inhibitor in Pseudomonas indigofera. J. Bacteriol. 131, 136-144 (1977).

29. Berg, I. A., Filatova, L. V. \& Ivanovsky, R. N. Inhibition of acetate and propionate assimilation by itaconate via propionyl-CoA carboxylase in isocitrate lyase-negative purple bacterium Rhodospirillum rubrum. FEMS Microbiol Lett. 216, 49-54 (2002).

30. Burg, J. M. et al. Large-scale bioprocess competitiveness: the potential of dynamic metabolic control in two-stage fermentations. Curr. Opin. Chem. Eng. 14, 121-136 (2016).
31. Kanamasa, S., Dwiarti, L., Okabe, M. \& Park, E. Y. Cloning and functional characterization of the cis-aconitic acid decarboxylase (CAD) gene from Aspergillus terreus. Appl Microbiol Biotechnol. 80, 223-229 (2008).

32. Elmore, J. R., Furches, A., Wolff, G. N., Gorday, K. \& Guss, A. M. Development of a high efficiency integration system and promoter library for rapid modification of Pseudomonas putida KT2440. Metab. Eng. Commun. 5, 1-8 (2017).

33. Tabor, S. \& Richardson, C. C. A bacteriophage T7 RNA polymerase/promoter system for controlled exclusive expression of specific genes. Proc. Natl Acad. Sci. USA 82, 1074-1078 (1985).

34. Wang, B., Barahona, M. \& Buck, M. Engineering modular and tunable genetic amplifiers for scaling transcriptional signals in cascaded gene networks. Nucleic Acids Res. 42, 9484-9492 (2014).

35. Hervas, A. B., Canosa, I. \& Santero, E. Transcriptome analysis of Pseudomonas putida in response to nitrogen availability. J. Bacteriol. 190, 416-420 (2008).

36. Hervas, A. B., Canosa, I., Little, R., Dixon, R. \& Santero, E. NtrC-dependent regulatory network for nitrogen assimilation in Pseudomonas putida. J. Bacteriol. 191, 6123-6135 (2009).

37. De Gier, J. W.\& Wagner, S. (Google Patents, 2012).

38. Moffatt, B. A. \& Studier, F. W. T7 lysozyme inhibits transcription by T7 RNA polymerase. Cell 49, 221-227 (1987)

39. Jones, J. A. et al. ePathOptimize: a combinatorial approach for transcriptional balancing of metabolic pathways. Sci. Rep. 5, 11301 (2015).

40. Salis, H. M., Mirsky, E. A. \& Voigt, C. A. Automated design of synthetic ribosome binding sites to control protein expression. Nat. Biotechnol. 27, 946-950 (2009).

41. Geiser, E. et al. Ustilago maydis produces itaconic acid via the unusual intermediate trans-aconitate. Micro. Biotechnol. 9, 116-126 (2016).

42. Gawron, O. \& Jones, L. Structural basis for aconitase activity inactivation by butanedione and binding of substrates and inhibitors. Biochim Biophys. Acta 484, 453-464 (1977).

43. Salvachua, D., Karp, E. M., Nimlos, C. T., Vardon, D. R. \& Beckham, G. T. Towards lignin consolidated bioprocessing: simultaneous lignin depolymerization and product generation by bacteria. Green. Chem. 17, 4951-4967 (2015).

44. Otten, A., Brocker, M. \& Bott, M. Metabolic engineering of Corynebacterium glutamicum for the production of itaconate. Metab. Eng. 30, 156-165 (2015)

45. Harder, B. J., Bettenbrock, K. \& Klamt, S. Temperature-dependent dynamic control of the TCA cycle increases volumetric productivity of itaconic acid production by Escherichia coli. Biotechnol. Bioeng. 115, 156-164 (2018).

46. Noh, M. H., Lim, H. G., Woo, S. H., Song, J. \& Jung, G. Y. Production of itaconic acid from acetate by engineering acid-tolerant Escherichia coli W. Biotechnol. Bioeng. 115, 729-738 (2018).

47. Jeon, H. G., Cheong, D. E., Han, Y., Song, J. J. \& Choi, J. H. Itaconic acid production from glycerol using Escherichia coli harboring a random synonymous codon-substituted 5 '-coding region variant of the cadA gene. Biotechnol. Bioeng. 113, 1504-1510 (2016).

48. Qi, L. S. et al. Repurposing CRISPR as an RNA-Guided Platform for Sequence-Specific Control of Gene Expression. Cell 152, 1173-1183 (2013).

49. Jayakody, L. et al. Thermochemical wastewater valorization via enhanced microbial toxicity tolerance. Energy Environ. Sci. 11, 1625-1638 (2018).

50. Elmore, J. R. et al. Engineered Pseudomonas putida simultaneously catabolizes five major components of corn stover lignocellulose: Glucose, xylose, arabinose, p-coumaric acid, and acetic acid. Metab. Eng. 62, 62-71 (2020).

51. Chen, X. W. et al. DMR (deacetylation and mechanical refining) processing of corn stover achieves high monomeric sugar concentrations (230 g L-1) during enzymatic hydrolysis and high ethanol concentrations (> 10\% v/v) during fermentation without hydrolysate purification or concentration. Energy Environ. Sci. 9, 1237-1245 (2016).

52. Marx, C. J. Development of a broad-host-range sacB-based vector for unmarked allelic exchange. BMC Res. Notes 1, 1 (2008).

53. Johnson, C. W. \& Beckham, G. T. Aromatic catabolic pathway selection for optimal production of pyruvate and lactate from lignin. Metab. Eng. 28, 240-247 (2015)

54. Love, M. I., Huber, W. \& Anders, S. Moderated estimation of fold change and dispersion for RNA-seq data with DESeq2. Genome Biology 15 (2014).

\section{Acknowledgements}

This work was authored in part by Oak Ridge National Laboratory, which is managed by UT-Battelle, LLC, for the U.S. DOE under contract DE-AC05-00OR22725. This work was also authored in part by Alliance for Sustainable Energy, LLC, the manager and operator of the National Renewable Energy Laboratory for the U.S. Department of Energy (DOE) under Contract No. DE-AC36-08GO28308, and in part by Battelle, the manager and operator of the Pacific Northwest National Laboratory for the U.S. Department of Energy under Contract No DE-AC05-76RLO1830. Funding was provided by the U.S. Department of Energy Office of Energy Efficiency and Renewable Energy BioEnergy Technologies Office. Research was in part sponsored by the Laboratory 
Directed Research and Development Programs at Oak Ridge National Laboratory (Project \#7866) and Pacific Northwest National Laboratory (Project \#207641).

\section{Author contributions}

J.R.E., A.M.G., D.S., and G.T.B. developed the concept. J.R.E., D.S., J.D.H., and G.L.P. designed experiments. G.N.D., J.M., J.R.E., and G.L.P. performed plasmid DNA construction, $P$. putida strain construction, and plate reader growth experiments. D.M.K. generated RNAseq libraries. E.A.H. performed bioinformatic analysis. D.J.P., J.R.E., and J.D.H. performed quantification of analytes in culture supernatants. C.S. and J.R.E. performed itaconic acid production assays. J.R.E., D.S., and J.H.B. performed data analysis. J.R.E., A.M.G., D.S., and G.T.B. wrote the manuscript.

\section{Competing interests}

J.R.E., J.D.H., D.S., G.T.B., and A.M.G. have patented technologies related to strains and pathways for the conversion of aromatics, sugars, and other feedstocks to itaconic acid under the title "Production of itaconic acid and related molecules from aromatic compounds” (U.S. Patent No. US10738333B2).

\section{Additional information}

Supplementary information The online version contains supplementary material available at https://doi.org/10.1038/s41467-021-22556-8.

Correspondence and requests for materials should be addressed to A.M.G.
Peer review information Nature Communications thanks Pablo Ivan Nikel and other, anonymous reviewers for their contributions to the peer review of this work.

Reprints and permission information is available at http://www.nature.com/reprints

Publisher's note Springer Nature remains neutral with regard to jurisdictional claims in published maps and institutional affiliations.

\section{(c) (i)}

Open Access This article is licensed under a Creative Commons Attribution 4.0 International License, which permits use, sharing, adaptation, distribution and reproduction in any medium or format, as long as you give appropriate credit to the original author(s) and the source, provide a link to the Creative Commons license, and indicate if changes were made. The images or other third party material in this article are included in the article's Creative Commons license, unless indicated otherwise in a credit line to the material. If material is not included in the article's Creative Commons license and your intended use is not permitted by statutory regulation or exceeds the permitted use, you will need to obtain permission directly from the copyright holder. To view a copy of this license, visit http://creativecommons.org/ licenses/by/4.0/.

(c) This is a U.S. Government work and not under copyright protection in the US; foreign copyright protection may apply 2021 